\title{
Anti-GRP78 autoantibodies induce endothelial cell activation and accelerate the development of atherosclerotic lesions
}

\author{
Elizabeth D. Crane, ${ }^{1}$ Ali A. Al-Hashimi, ${ }^{2,3}$ Jack Chen, ${ }^{2}$ Edward C. Lynn, ${ }^{2}$ Kevin Doyoon Won, ${ }^{2}$ \\ Šárka Lhoták, ${ }^{2}$ Magda Naeim, ${ }^{2}$ Khrystyna Platko, ${ }^{2}$ Paul Lebeau, ${ }^{2}$ Jae Hyun Byun, ${ }^{2}$ \\ Bobby Shayegan, ${ }^{3}$ Joan C. Krepinsky, ${ }^{2}$ Katey J. Rayner, ${ }^{4}$ Serena Marchiò,, ${ }^{5,6}$ Renata Pasqualini, ${ }^{7,8}$ \\ Wadih Arap, ${ }^{7,9}$ and Richard C. Austin ${ }^{1,2}$ \\ 'Department of Biochemistry and Biomedical Sciences, ${ }^{2}$ Department of Medicine, Division of Nephrology, and ${ }^{3}$ Division \\ of Urology, Department of Surgery, McMaster University and The Research Institute of St. Joe's Hamilton, Hamilton, \\ Ontario, Canada. ${ }^{4}$ Department of Biochemistry, Microbiology, and Immunology, Faculty of Medicine, University of \\ Ottawa and University of Ottawa Heart Institute, Ottawa, Ontario, Canada. ${ }^{5}$ Department of Oncology, University of Turin, \\ Candiolo, Italy. ${ }^{6}$ Candiolo Cancer Institute - Fondazione del Piemonte per l'Oncologia, Istituto di Ricerca e Cura a Carattere \\ Scientifico, Candiolo, Italy. ${ }^{7}$ Rutgers Cancer Institute of New Jersey, Newark, New Jersey, USA. ${ }^{8}$ Division of Cancer Biology, \\ Department of Radiation Oncology, and ${ }^{9}$ Division of Hematology/Oncology, Department of Medicine, Rutgers New Jersey \\ Medical School, Newark, New Jersey, USA.
}

Authorship note: EDC and AAAH are co-first authors.

Conflict of interest: RCA and JCK receive biomedical research funding from Amgen Canada. RP and WA founded and hold equity in Ablaris Therapeutics, Alvos Therapeutics, AMP Pharmaceuticals, Ceramide Therapeutics, MBrace Therapeutics, and PhageNova Bio. RP is Chief Scientific Officer and a paid consultant for PhageNova Bio. $\mathrm{RP}$ and WA are inventors on patent applications (PCT/US2017/052661, 20180256740) for related technologies and are entitled to standard royalties if commercialization occurs. Rutgers, the State University of New Jersey, currently manages these arrangements in accordance with its established institutional conflict-of-interest policy.

License: Copyright 2018, American Society for Clinical Investigation.

Submitted: December 19, 2017 Accepted: November 9, 2018 Published: December 20, 2018

\section{Reference information:}

JCI Insight. 2018;3(24):e99363. https://doi.org/10.1172/jci.

insight.99363.
The 78-kDa glucose-regulated protein (GRP78) is an ER molecular chaperone that aids in protein folding and secretion. However, pathological conditions that cause ER stress can promote the relocalization of GRP78 to the cell surface (csCRP78), where it acts as a signaling receptor to promote cancer progression. csGRP78 also possesses antigenic properties, leading to the production of anti-GRP78 autoantibodies, which contribute to tumor growth. In contrast, the presence and role of anti-GRP78 autoantibodies in atherosclerosis is unknown. Here, we show that atheroscleroticprone $\mathrm{ApoE}^{-/-}$mice develop circulating anti-GRP78 autoantibodies that bind to csGRP78 on lesionresident endothelial cells. Moreover, GRP78-immunized $\mathrm{ApoE}^{-/-}$mice exhibit a marked increase in circulating anti-GRP78 autoantibody titers that correlated with accelerated lesion growth. Mechanistically, engagement of anti-CRP78 autoantibodies with csCRP78 on human endothelial cells activated NF- $\mathrm{KB}$, thereby inducing the expression of ICAM-1 and VCAM-1, a process blocked by NF- $\kappa B$ inhibitors. Disrupting the autoantibody/csGRP78 complex with enoxaparin, a low-molecularweight heparin, reduced the expression of adhesion molecules and attenuated lesion growth. In conclusion, anti-GRP78 autoantibodies play a crucial role in atherosclerosis development, and disruption of the interaction between anti-GRP78 autoantibodies and csGRP78 represents a therapeutic strategy.

\section{Introduction}

Atherosclerotic lesion formation, progression, and plaque rupture are the underlying causes of cardiovascular disease (CVD) in the developed world $(1,2)$. In a healthy, unobstructed blood vessel, endothelial cells (ECs) line the vascular wall and act as the first layer of defense against vascular damage and immune cell recruitment that contributes to the early stages of atherosclerosis. EC dysfunction, induced by mechanical strain, local inflammation, and/or the uptake of modified lipoproteins is a major contributing factor to the initiation and growth of atherosclerotic lesions (1). Molecular and physiologic changes to the vascular endothelium, including secretion of proinflammatory mediators and recruitment of monocytes into the blood vessel wall are important contributors to the early molecular and cellular events in atherogenesis (2). Moreover, enhanced expression of adhesion molecules ICAM-1 and VCAM-1 on the surface of lesion-resident ECs is crucial for the capture and transmigration of leukocytes into the subendothelial space of developing atherosclerotic lesions (1).

As a means of characterizing unique surface proteins present on atherosclerotic lesions in vivo, Liu et al. screened a phage-display random peptide library and identified csGRP78 (3). Although GRP78 was observed on the surface of lesion-resident ECs in the aortas from $A p o E^{-1 /}$ mice and in human lesions from 
iliac artery segments, a causal role for csGRP78 in lesion development has not as yet been established (3). Studies investigating the function of csGRP78 in cancer cell proliferation and tumor growth have reported that it acts as a membrane-associated receptor (4), thereby mediating a variety of signaling pathways through its association with assorted ligands and coreceptors (5). Recent evidence has highlighted the role of csGRP78 as a regulator of cancer cell survival and proliferation upon interaction with $\alpha_{2}$-macroglobulin through PI3K/AKT signaling (6), while conversely highlighting a role for csGRP78 in inducing apoptosis through suppression of Ras/MAPK when bound by extracellular Par-4 (7). Our own recent findings have demonstrated the ability of csGRP78 to activate tissue factor procoagulant activity by mediating $\mathrm{Ca}^{2+}$ release from the ER via PLC-mediated $\mathrm{IP}_{3}$ production $(8,9)$. It remains unknown, however, whether csGRP78 plays a role in atherosclerotic lesion growth and progression.

While the precise molecular mechanism by which GRP78 translocates to the cell surface remains unclear (10), it has recently been shown that induction of ER stress may actually stimulate such relocalization (11). Given that ER stress is a known hallmark of atherosclerotic lesion progression (12-14), a link between csGRP78 in ECs and atherogenesis is tantalizingly plausible. The presence of csGRP78 can also induce the production of anti-GRP78 autoantibodies by the humoral immune system (15). Autoantibodies to GRP78 have been identified circulating in the peripheral blood of patients with a variety of cancers, including melanoma, prostate cancer, and ovarian cancer (15). Although anti-GRP78 autoantibodies that recognize both the $\mathrm{N}$ - and $\mathrm{C}$-terminal regions of GRP78 have been found, it is important to note that the majority of the anti-GRP78 autoantibodies found in the human circulation are directed against a specific epitope on the N-terminus of surface GRP78 $\left(\mathrm{Leu}_{98}-\mathrm{Leu}_{115}\right)$, indicating the relevance of this particular antigenic region (16). Moreover, high levels of these anti-GRP78 autoantibodies in patients correlate with advanced disease and poor survival outcomes, at least in patients with prostate cancer (15). In addition, we have demonstrated the presence of an anti-GRP78 autoantibody/csGRP78 molecular circuit in bladder carcinoma cells (8) and, more recently, in prostate cancer (9). Furthermore, the low-molecular-weight heparin (LMWH) enoxaparin (17) binds to csGRP78, thereby disrupting the engagement of anti-GRP78 autoantibodies and attenuating their effect on increased rate of tumor growth (9).

The correlation between anti-GRP78 autoantibodies and atherosclerosis and whether the mechanism observed in cancer is relevant to atherosclerotic lesion development have not yet been examined. Here, we show that induction of ER stress results in increased amounts of csGRP78 in cultured human aortic ECs (HAECs). Moreover, we identify anti-GRP78 autoantibodies specific to the N-terminus of csGRP78 in the $A p o E^{-/-}$mouse model of atherosclerosis and demonstrate that autoantibody levels correspond to atherosclerotic lesion progression; in addition, activation of csGRP78 by anti-GRP78 autoantibodies induces proinflammatory mediators and adhesion molecule expression in HAECs expressing csGRP78. Finally, increasing the circulating levels of anti-GRP78 autoantibodies accelerates atherosclerotic lesion growth in $A p o E^{-1-}$ mice through a direct activation of csGRP78 on lesion-resident ECs, an effect that is attenuated by enoxaparin. These findings provide a solid foundation for understanding the role of anti-GRP78 autoantibodies and the activation of csGRP78 in EC function during atherogenesis.

\section{Results}

The development of atherosclerotic lesions correlates with increased anti-GRP78 autoantibody titers. Mice with atherosclerotic lesions exhibit increased levels of anti-GRP78 autoantibodies. High levels of anti-GRP78 autoantibodies identified in prostate cancer patients were shown to correlate with accelerated cancer progression and reduced overall survival (15). Although csGRP78 has been identified on atherosclerotic lesion-resident ECs in mice and humans (3), the role of anti-GRP78 autoantibodies was not assessed in this pathological setting. To investigate whether anti-GRP78 autoantibody levels correlate with accelerated lesion development, repeated blood draws from $A p o E^{-/-}$and control C57BL/ 6 mice were taken between 8 and 20 weeks of age, a marked period of lesion growth in $A p o E^{-/-}$mice but not for otherwise isogenic C57BL/6 wild-type control mice. We observed that anti-GRP78 autoantibody titers increased with age in $A p o E^{-/-}$mice fed a chow diet and were much higher than levels in C57BL/ 6 mice at 20 weeks of age (Figure 1A). To examine whether the timing of lesion development could influence anti-GRP78 autoantibody titers, mice were fed a high-fat diet (HFD) that markedly accelerates atherosclerosis progression. Apo $E^{-/-}$mice on a HFD showed much higher titers of anti-GRP78 autoantibodies compared with C57BL/6 mice at 17 weeks of age (Figure 1B), suggesting a more rapid induction of anti-GRP78 autoantibodies under dietary conditions that induce atherosclerosis. In contrast, 4-PBA, a small chemical chaperone known to reduce ER stress and lesion 
development $(18,19)$, blocked atherosclerotic lesion growth and reduced anti-GRP78 autoantibody titers in $A p o E^{-1-}$ mice (Supplemental Figure 1; supplemental material available online with this article; https:// doi.org/10.1172/jci.insight.99363DS1).

Anti-GRP78 autoantibodies bind to atherosclerotic lesion-resident ECs in ApoE ${ }^{-/-}$mice. To determine whether anti-GRP78 autoantibodies interact with lesion-resident ECs, 18-week-old $A p o E^{-/-}$mice were administered i.v. via the tail vein with biotinylated anti-GRP78 autoantibodies (b-anti-GRP78 autoantibodies) or biotinylated mouse IgG (b-IgG) and euthanized after 30 minutes. The ascending aorta and proximal arch were dissected and double-stained en face for ECs (anti-CD31) and for the presence of biotinylated antibodies (Alexa Fluor 594 Streptavidin). Binding of b-anti-GRP78 autoantibodies was assessed in regions with high probability (HP) and low probability (LP) of developing lesions (Figure 1C), as described by liyama et al. (20). We observed that b-anti-GRP78 autoantibodies bound to lesion-resident ECs in HP regions (Figure 1D; top middle) but not in LP regions (Figure 1D; left). To confirm the binding specificity, no signal was detected in either LP or HP regions from mice receiving b-IgG (Figure 1D; bottom) or in HP regions incubated with secondary antibodies only (Figure 1D; top right). These results provide evidence that anti-GRP78 autoantibodies directly interact with atherosclerotic lesion-resident ECs in $A p o E^{-/-}$mice. Finally, we measured anti-GRP78 autoantibody titers in patients with established CVD and compared them to age-matched control patients without CVD. Similar to our in vivo studies, CVD patients had significantly elevated levels of anti-GRP78 autoantibody titers when compared with healthy, aged-matched controls (Figure 1E). These data provide clear evidence that autoantibody titers correlate with atherosclerotic lesion development both in mice and in humans and that anti-GRP78 autoantibodies bind csGRP78 on the surface of lesion-resident ECs.

Elevated levels of anti-GRP78 autoantibodies exacerbate atherosclerosis in ApoE $E^{-1}$ mice. In order to increase the levels of circulating anti-GRP78 autoantibodies in vivo and determine whether they play a role in lesion development, 6-week-old $A p o E^{-/-}$mice on a chow diet were immunized with recombinant GRP78 (rGRP78) or control ovalbumin (OVA). ApoE $E^{-/-}$mice receiving rGRP78 produced much higher levels of anti-GRP78 autoantibodies at both 12 and 15 weeks of age compared with control OVA-immunized mice (Figure 2A). Atherosclerotic lesion development was measured by histologic quantification of lesion size at the aortic root as described previously (21). Mice with higher anti-GRP78 autoantibody titers (rGRP78 immunized) had much larger lesions compared with control OVA-immunized mice (Figure 2, B and C). Quantification of the necrotic area in lesions demonstrated a much greater degree of necrosis in mice with higher levels of anti-GRP78 autoantibodies (Figure 2D). To further characterize the progression of atherosclerosis, lesions were scored based on criteria described by van Vlijmen et al. (22) and assigned to 3 categories: (a) early lesions consisting of only fatty streaks containing foam cells; (b) moderate lesions characterized by extension into the media and mild fibrosis; and (c) advanced lesions containing cholesterol clefts and necrotic area deeper into the media. Mice with higher anti-GRP78 autoantibody titers had more advanced lesions and fewer early lesions compared with control OVA-immunized mice ( $30 \%$ vs. $17 \%$ and $29 \%$ vs. $44 \%$, respectively, Figure 2E). Plasma triglyceride (Figure $2 \mathrm{~F}$ ) and total cholesterol (Figure 2G) levels were not different between the groups, indicating that differences in lesion growth and severity are not the result of alterations in circulating lipid levels. Taken together, these results indicate that elevated levels of anti-GRP78 autoantibodies contribute to atherosclerotic lesion development.

ER stress inducers increase of the levels of cSGRP78 on ECs. Previous reports have shown that ER stress is sufficient to cause cell-surface expression of GRP78 in cancer cell lines $(11,23)$. Consistent with these findings, primary HAECs treated with thapsigargin (Tg; $300 \mathrm{nM}$ ) or tunicamycin (Tm; $2.5 \mu \mathrm{g} / \mathrm{ml}$ ) for 16, 24, or 32 hours exhibited increased levels of GRP78 in surface fractions (sGRP78). This increase peaked at 16 and 24 hours after induction of ER stress (Figure 3, A and B). Consistent with previous studies, CD31, a surface marker of ECs, is downregulated under conditions of ER stress (24). The induction of ER stress by both Tg and Tm in HAECs was confirmed by the increased protein expression of total GRP78, IRE1 $\alpha$, and p-eIF1 $\alpha$. To investigate whether physiologically relevant factors could stimulate csGRP78 expression in ECs, HAECs were exposed to well-established natural inducers of ER stress, including lipid oxidation products, 7-ketocholesterol and 4-HNE (25), as well as to the inducers of oxidative stress, Sin-1 and peroxynitrite (26). Increased csGRP78 expression was also observed under these conditions (Figure 3C). Consistent with these in vitro results and our previous findings $(12,13)$, early atherosclerotic lesions from chow-fed 12 -week-old $A p o E^{-/}$mice, defined as fatty streaks (Supplemental Figure 2), showed the presence of physiological inducers of ER stress, including free cholesterol (filipin staining) and peroxynitrite (3-nitrotyrosine staining) (Supplemental Figure 3), as well as increased expression of several ER stress markers, including GRP78 itself, calreticulin, CHOP, pPERK, and TDAG51 in atherosclerotic lesion-resident macrophages and ECs (Supplemental Figure 4). 
A

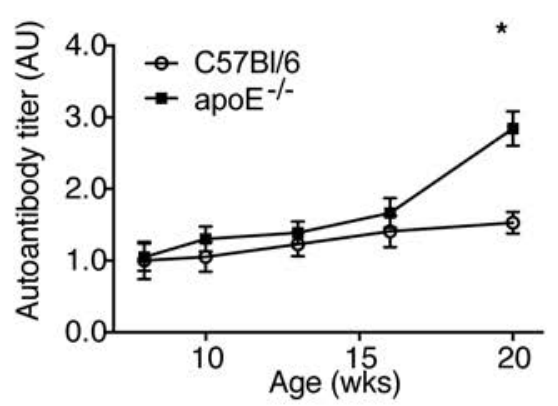

B

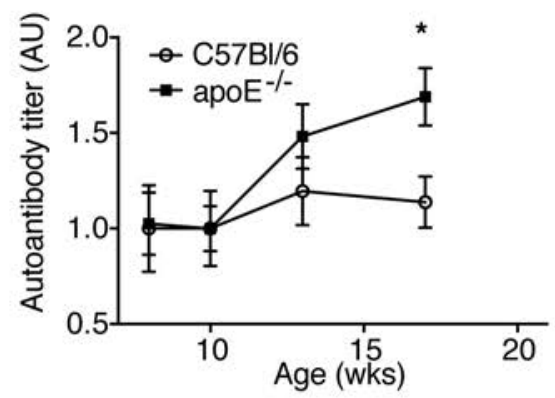

C

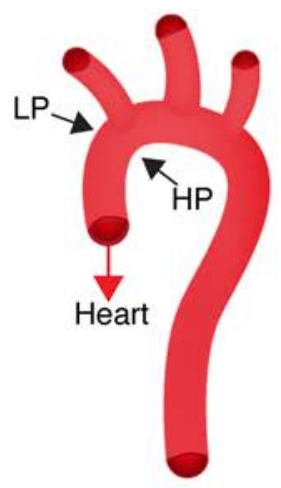

E

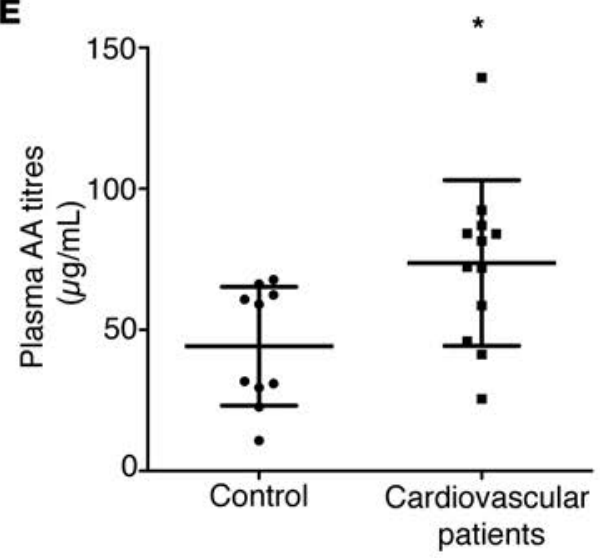

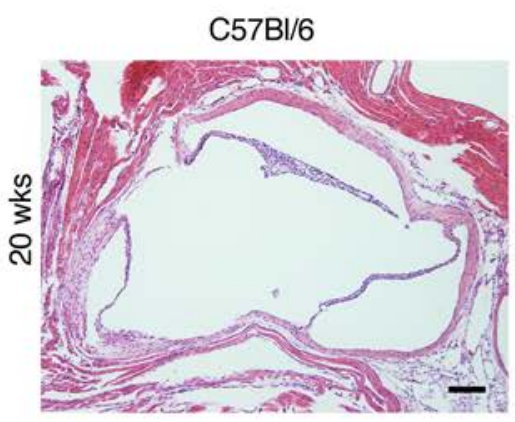
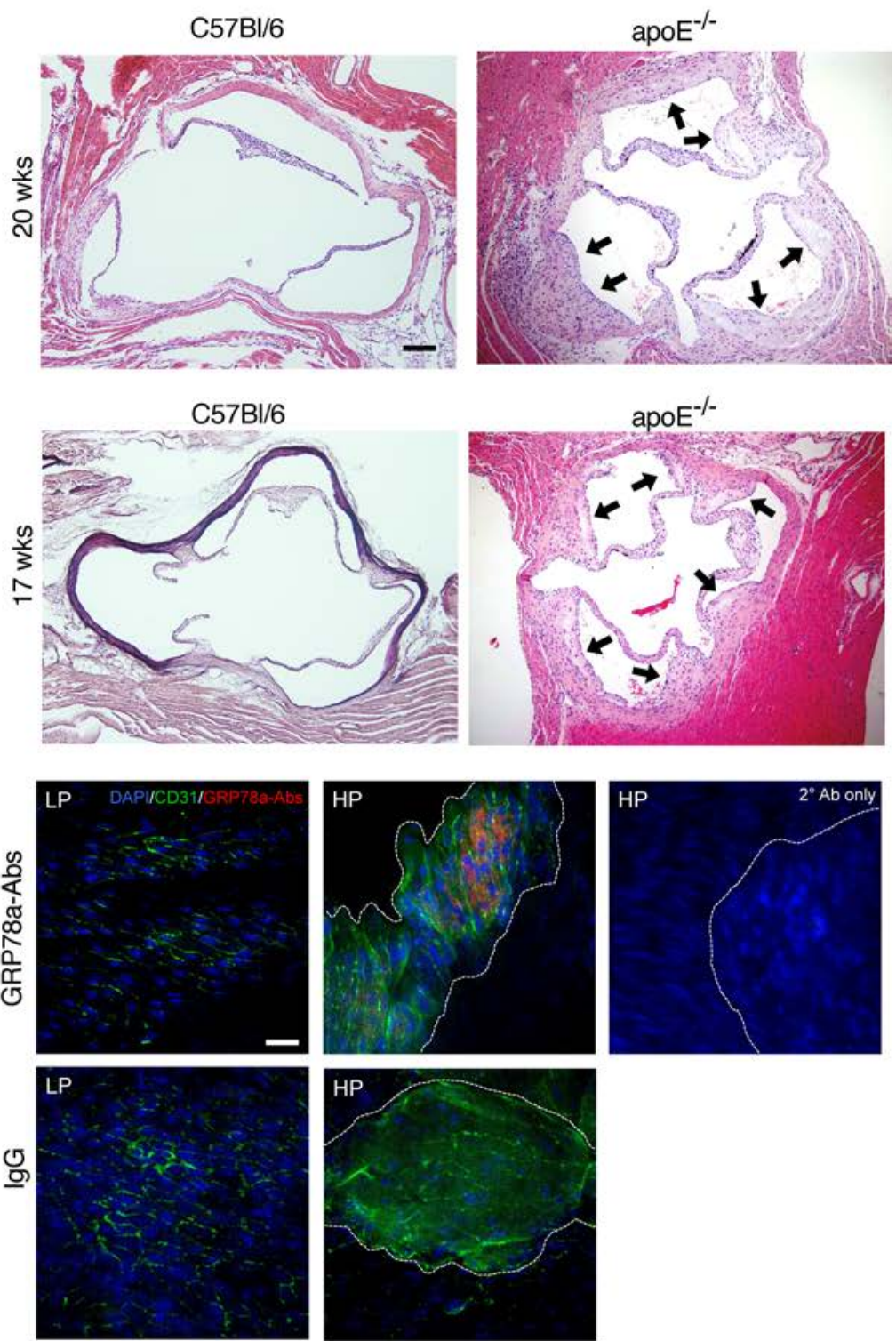

Figure 1. Anti-GRP78 autoantibodies are elevated in $\mathrm{ApoE}^{-/-}$mice. Serum levels of antiGRP78 autoantibodies were measured by ELISA in female C57BL/ 6 and $A p o E^{-/-}$mice on either a chow (A) or HFD (B) ( $n=7-9$ per group). ${ }^{*} P<0.05$ versus age-matched C57BL/6 mice, 2 -tailed $t$ test. All data are presented as a ratio of absorbance units (AU) relative to a standard sample. Representative images of aortic root sections from mice in $\mathbf{A}$ and B are presented; arrows indicate atherosclerotic lesions. Original magnification, $\times 10$. (C) Location of LP and HP regions on the ascending aorta and proximal arch assessed for atherosclerotic lesions and presence of bound anti-GRP78 autoantibodies. (D) Representative immunofluorescence images of anti-GRP78 autoantibody binding to lesion-resident ECs in the aortic arch of $A p o E^{-1-}$ mice. Mice were injected i.v. with $10 \mu \mathrm{g}$ b-anti-GRP78 autoantibodies or b-IgG and euthanized 30 minutes after injection. Multiple pieces from LP and HP regions of the arch were dissected for each mouse and stained en face, and 3 images per region were qualitatively assessed for the presence/absence of anti-GRP78 autoantibody or IgG staining in lesion and nonlesion areas. Dashed lines indicate lesion areas. ECs are stained in green (rat anti-mouse CD-31) and anti-GRP78 autoantibodies or IgG are stained in red (streptavidin-conjugated secondary antibody). Nuclei are counterstained with DAPI (blue). One anti-GRP78 autoantibody and one b-IgG mouse were injected in parallel for each experiment. Original magnification, $\times 40$. (E) Anti-GRP78 autoantibody titers in a clinical population with established CVD and age-matched controls, as measured by ELISA $(n=12) .{ }^{*} P<0.05$ versus age-matched controls, 2 -tailed $t$ test. 
A

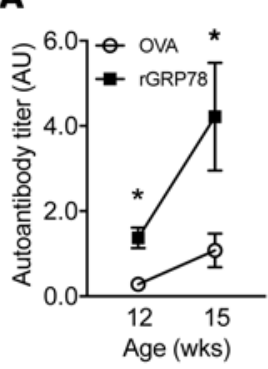

D

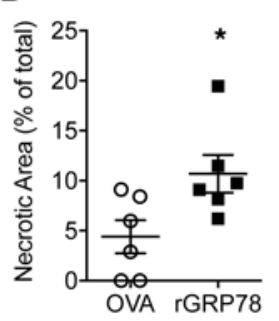

B

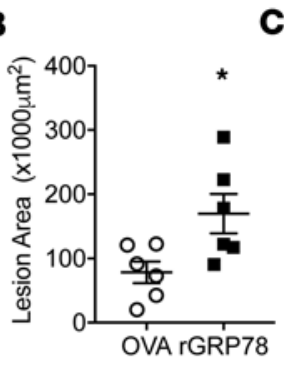

E

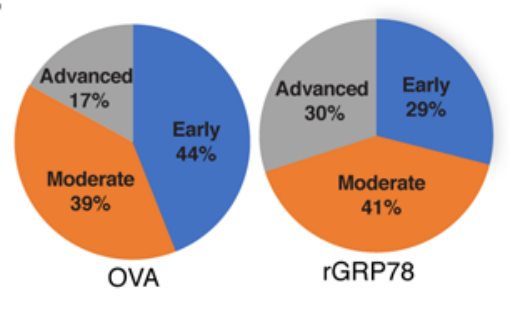

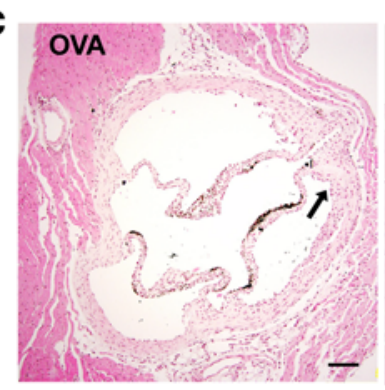

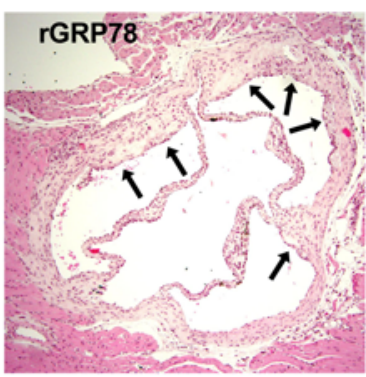

$\mathbf{F}$

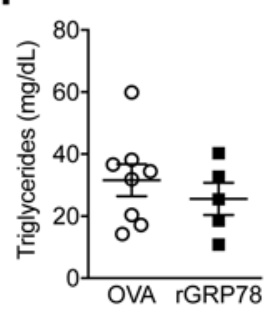

G

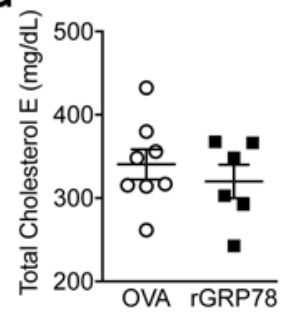

Figure 2. Atherosclerotic lesion growth is accelerated in ApoE ${ }^{-/-}$mice with elevated anti-GRP78 autoantibody titers. (A) Serum levels of anti-GRP78 autoantibodies were measured by ELISA in female $A p o E^{-/-}$mice fed a chow diet and injected 3 times at 10-day intervals with $50 \mu \mathrm{g} / \mathrm{ml}$ OVA or rCRP78 beginning at 6 weeks of age. Data are presented as a ratio of absorbance units (AU) relative to a standard sample $\left(n=6\right.$ per group). ${ }^{*} P<0.05$ versus 0 VA at each time point, 2-tailed $t$ test. (B) Quantification of atherosclerotic lesion size at the aortic root from 15 -week-old immunized mice. Lesion size was measured in 5-6 sections at 80- $\mu$ m intervals ( $n=6$ per group). ${ }^{*} P<0.01$ versus OVA immunized mice, 2 -tailed $t$ test. (C) Representative cross-section images of the aortic root stained with $\mathrm{H} \& \mathrm{E}$ for each treatment group. Arrows indicate atherosclerotic lesions. Original magnification, $\times 10$. Scale bar: $100 \mu \mathrm{m}$. (D) Quantification of necrotic area in lesions at the aortic root. Data are expressed as percentage of total lesion area ( $n=6$ per group). ${ }^{*} P<0.01$ versus OVA treated mice, 2-tailed $t$ test. (E) Classification of atherosclerotic lesions in ApoE-1- mice immunized with OVA or rGRP78. Plasma levels of (F) triglycerides and $(\mathbf{G})$ total cholesterol were measured in immunized $A p o E^{-/-}$mice at 15 weeks of age by colorimetric assays ( $n=6-8$ per group).

Anti-GRP78 autoantibodies increase adhesion molecule expression and induce leukocyte adhesion. Given that anti-GRP78 autoantibody titers increase during atherosclerotic lesion development, we investigated whether anti-GRP78 autoantibodies trigger a proatherogenic response in cultured ECs. Figure 4A demonstrates that anti-GRP78 autoantibodies can induce the expression of ICAM1 and VCAM1 in a dose-dependent manner. To clearly delineate the role of anti-GRP78 autoantibodies on increased expression of these adhesion molecules, HAECs were pretreated with Tm for 24 hours to increase csGRP78 expression and subsequently exposed to anti-GRP78 autoantibodies. Treatment with anti-GRP78 autoantibodies resulted in much higher mRNA expression of ICAM1 and VCAM1 compared with Tm or IgG controls (Figure 4B). Furthermore, incubation of anti-GRP78 autoantibodies with a synthetic conformational peptide (CP; sequence CNVSKDSC) $(8,15)$ that binds anti-GRP78 autoantibodies and blocks signaling of csGRP78 clearly reduced ICAM1 and VCAM1 expression (Figure 4B). Our studies with prostate cancer showed that enoxaparin (27), an FDA-approved low-molecular-weight heparin anticoagulant, can inhibit the binding of anti-GRP78 autoantibody to csGRP78. Likewise, enoxaparin was effective in specifically blocking the increased expression of ICAM1 and VCAM1 in HAECs elicited by the anti-GRP78 autoantibodies (Figure 4C). Expression of cell adhesion molecules on ECs is critical for monocyte recruitment into atherosclerotic lesions $(28,29)$. Therefore, we examined whether the anti-GRP78 autoantibody-induced increase in ICAM1 and VCAM1 expression affected the adhesion of human monocyte-derived cells (U937) to HAECs. Treatment of HAECs with Tm followed by anti-GRP78 autoantibodies markedly increased the number of attached U937 cells compared with control isogenic IgG-treated cells (Figure 4D). Treatment with Tm or anti-GRP78 autoantibodies alone had no effect on U937 cell attachment. Consistent with the effects seen on adhesion molecule expression, addition of the CP specifically reduced U937 attachment to HAECs. As expected, TNF- $\alpha$ treatment clearly increased U937 attachment to HAECs.

Anti-GRP78 autoantibodies promote proinflammatory gene expression and adhesion molecule expression via the $N F-\kappa B$ pathway. To determine potential pathways activated by anti-GRP78 autoantibodies, we utilized the NanoString technology. Examining ECs treated with anti-GRP78 autoantibodies revealed that inflammatory cytokines are upregulated (Figure 5A). Inflammation is directly linked to cell adhesion molecule 
A $\operatorname{Tg}(300 \mathrm{nM})$

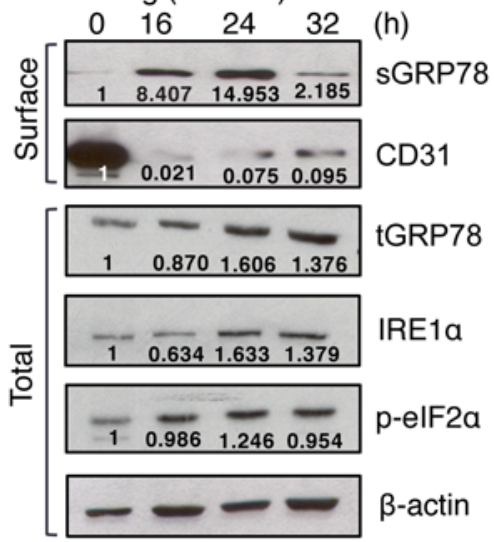

B

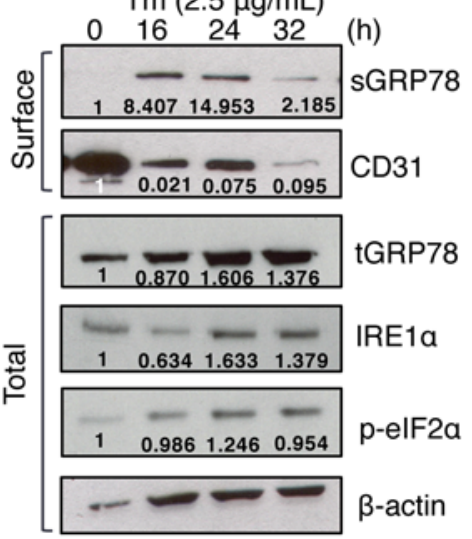

C

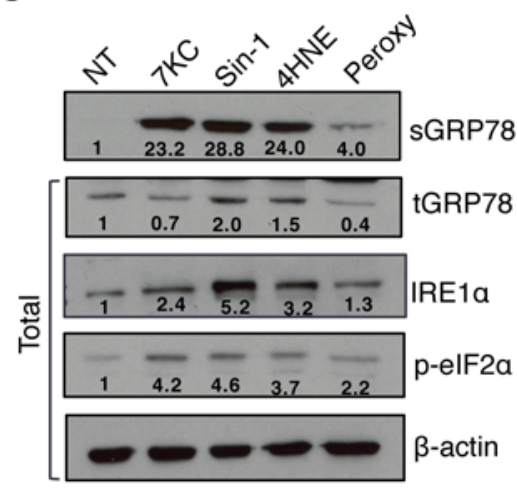

Figure 3. ER stress causes an increase in csGRP78 levels on ECs. HAECs were treated with (A) $2.5 \mu \mathrm{g} / \mathrm{ml} \mathrm{Tm}$ or (B) $300 \mathrm{nM}$ Tg for the indicated time period or with (C) 7-ketocholesterol (7KC), Sin-1, 4-hydroxynonenal (4HNE), or peroxynitrite (Peroxy) for 8 hours, after which surface proteins were biotinylated and separated by streptavidin pull down. Total cell lysates (tGRP78) and surface protein fractions (sGRP78) were subjected to Western blotting for detection of GRP78, the EC marker CD31, IRE1 $\alpha$, or phospho-elF1 $\alpha$. Blots were also immunostained for $\beta$ actin as a protein loading control. Densitometry quantification is indicated under each band relative to untreated cells and normalized to $\beta$-actin.

expression in ECs (30), and this is regulated by the NF- $\mathrm{BB}$ signaling pathway (31). To delineate the contribution of NF- $\mathrm{KB}$ to the activation of ECs by anti-GRP78 autoantibodies, we examined the expression of a panel of cytokines and chemokines known to be induced by NF- $\mathrm{KB}$. In addition to treating cells with antiGRP78 autoantibodies, a cotreatment strategy with NF- $\mathrm{B}$ inhibitors (TPCA-1, BAY 11-7082, SC-514, JSH-23) was conducted and mRNA expression was measured by quantitative RT-PCR (8). mRNA levels of VCAM1 (Figure 5B), ICAM1 (Figure 5C), cytokines IL-1 $\beta$ (Figure 5D) and IL-6 (Figure 5E), and the chemokine MIP-1 $\alpha$ (Figure 5F) were much higher in anti-GRP78 autoantibody-treated HAECs compared with IgG-treated cells. Pretreatment of cells with these various NF- $\kappa$ B inhibitors was shown to decrease the expression of cell adhesion molecules, cytokines, and chemokines (Figure 5, B-F). To confirm that $\mathrm{NF}-\kappa \mathrm{B}$ plays a role in anti-GRP78 autoantibody-mediated signaling, ECs expressing csGRP78 were pretreated with the NF- $\mathrm{BB}$ activation inhibitor II, JSH-23, for 2 hours followed by anti-GRP78 autoantibodies or human IgG. NF- $\mathrm{BB}$ inhibition reduced anti-GRP78 autoantibody-mediated induction of ICAM-1 and VCAM-1 expression (Figure 5, B and C). In addition, treatment with JSH-23 reduced monocyte adhesion to HAECs treated with anti-GRP78 autoantibodies (Figure 5G), further supporting the functional involvement of the NF- $\mathrm{kB}$ pathway in the anti-GRP78 autoantibody/csGRP78 molecular circuit. Consistent with these in vitro findings, the expression of p65 subunit of $\mathrm{NF}-\kappa \mathrm{B}$ was noticeably increased in atherosclerotic sections from $A p o E^{-/-}$mice immunized with full-length, functionally active rGRP78 compared with control mice immunized with OVA (Supplemental Figure 5).

Enoxaparin or TPCA-1 inhibits the effect of anti-GRP78 autoantibody on NF- $\mathrm{B}$ p65 subunit nuclear translocation. Our data indicate that NF- $\mathrm{BB}$ activation may be required for anti-GRP78 autoantibody-induced expression of proinflammatory markers in HAECs. To further investigate this phenomenon, we evaluated the localization of the NF- $\mathrm{kB}$ p65 subunit within the ECs by immunofluorescence. Similar to the TNF- $\alpha-$ positive control for NF- $\mathrm{BB}$ activation, treatment of HAECs with anti-GRP78 autoantibodies elicited nuclear translocation of the p65 subunit (Figure 6A), a hallmark of NF- $\mathrm{kB}$ transcriptional activation. However, this finding was reversed when cells were pretreated with enoxaparin or TPCA-1 (Figure 6, A and B).

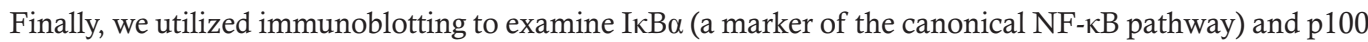
(a marker of the noncanonical NF- $\mathrm{B}$ pathway) expression following anti-GRP78 autoantibody treatment (Figure 6C). A decrease in the protein expression of $\mathrm{I} \kappa \mathrm{B} \alpha$, but not $\mathrm{p} 100$, was observed after anti-GRP78 autoantibody treatment (Figure 6C), suggesting that the NF- $\mathrm{kB}$ canonical pathway is the main driver of this activation; TNF- $\alpha$ treatment served as a positive control as described previously (29).

Direct injection of anti-GRP78 autoantibodies into ApoE $E^{-/-}$mice accelerates atherosclerotic lesion growth. To directly elucidate the effect of anti-GRP78 autoantibodies on the rate of lesion growth in mice, we injected $A p o E^{-/-}$mice with patient-purified anti-GRP78 autoantibodies to achieve a pathologic dose of $60 \mu \mathrm{g} /$ 
$\mathrm{ml}(9,16)$. Enoxaparin cotreatment, used as an intervention approach, reversed the positive effect of the anti-GRP78 autoantibodies on lesion growth. Representative images of the treatment groups (Figure 7, $\mathrm{A}$ and $\mathrm{B}$ ), along with quantification of plaque volume for all the acquired images (Figure 7C), are shown. We further evaluated NF- $\mathrm{B}$ activation by identifying ECs with positive $\mathrm{p} 65$ subunit nuclear staining. Only the anti-GRP78 autoantibody-treated group exhibited enhanced p65 subunit-positive nuclei staining in atherosclerotic lesion-resident ECs (Figure 7D). In support of our cell culture studies, enoxaparin reduced p65 immunostaining of lesion-resident ECs (Figure 7D, AA+Enox). These findings suggest that the direct i.v. administration of anti-GRP78 autoantibodies in mice promotes atherosclerotic lesion progression, a pathological process specifically mitigated by enoxaparin.

\section{Discussion}

Atherosclerosis contributes to the development of cardiovascular pathologies that can lead to serious complications, including stroke or myocardial infarction $(2,32)$. Developing a sound understanding of the mechanism of atherosclerotic lesion growth and plaque stability provides important insights for designing new therapeutics that can decrease the burden of human CVD and its complications. This study provides compelling evidence for the role of an anti-GRP78 autoantibody/csGRP78 signaling circuit on ECs as drivers of atherosclerotic lesion growth and possibly plaque rupture. Under pathological conditions, such as atherosclerosis and cancer, GRP78 has been identified on the surface of many cell types, but the precise cellular events that allow its evasion from ER retention mechanisms and consequent translocation to the plasma membrane are not entirely understood. GRP78 possesses the classic C-terminal Lys-Asp-Glu-Leu (KDEL) sequence that is recognized by the KDEL receptor on the ER lipid bilayer, thereby resulting in selective retrograde transport back to the ER from the Golgi $(33,34)$. One possible explanation is that the KDEL receptor is overwhelmed as a result of its saturation due to chronic or acute ER stress, allowing proteins containing the KDEL sequence to escape to the cell surface (35, 36). It is also possible that conditions of ER stress modify the structure of GRP78 or its interaction with specific binding partners that would allow for its movement from the ER to the cell surface. GRP78 was first identified on the surface of lesion-resident ECs by Liu et al. (3) by using a phage-display peptide library to screen for novel surface markers specifically present on atherosclerotic lesions. While this was the first indication that GRP78 might have an alternative role in atherosclerosis other than its conventional role in ER stress and the UPR per se, the underlying cellular mechanism by which csGRP78 modulates atherogenesis as well as the detection and effect of circulating anti-GRP78 autoantibodies was not investigated.

Our previous studies show that csGRP78 signaling promotes cancer cell proliferation (8) and accelerates tumor growth (9), which raised the question as to whether activation of csGPR78 via anti-GRP78 autoantibodies can also contribute to atherosclerotic lesion development and progression. We systematically investigated how this interaction could modulate EC function and atherosclerotic lesion development in $A p o E^{-/-}$mice (summarized in Figure 8). The observation that $A p o E^{-/-}$mice, a well-established mouse model of atherosclerosis $(13,19)$, have elevated anti-GRP78 autoantibody titers that correlate with lesion growth, compared with age-matched C57BL/ 6 mice, suggests a link between the progression of atherosclerotic lesions and anti-GRP78 autoantibody levels. In support of these findings, we observed that the acceleration in atherosclerotic lesion growth correlated with an increase in autoantibody titers in $A p o E^{-/}$mice placed on a HFD compared with chow-fed $A p o E^{-/-}$mice. The association between anti-GRP78 autoantibodies and atherosclerotic lesion development supports the concept that anti-GRP78 autoantibodies modulate and accelerate the formation of advanced lesions. Further, although C57BL/ 6 mice on the chow diet do not develop atherosclerosis when fed a HFD, these mice demonstrated detectable levels of circulating anti-GRP78 autoantibodies. Thus, it is possible that csGRP78 is expressed on ECs within fatty streaks of C57BL/6 mice, thereby contributing to the detectable levels of GRP78 autoantibodies reported here. Clinically, increased levels of anti-GRP78 autoantibodies were detected in a cohort of patients with established CVD, compared with healthy, agedmatched controls. This clinical observation parallels our in vivo findings and hint to the idea that a threshold level of circulating anti-GRP78 autoantibodies is required to elicit csGRP78-mediated EC activation. Indeed, a threshold of anti-GRP78 autoantibodies is required for tumor cell proliferation (8) and growth (9); additionally, these autoantibody levels in mice correspond to titers seen in patients $(15,16)$.

Additional evidence to indicate a role of anti-GRP78 autoantibodies in atherosclerosis was demonstrated by the direct binding of anti-GRP78 autoantibodies to lesion-resident ECs within the aortic root. No detectable binding of these autoantibodies was observed on the surface of ECs distal to the atherosclerotic lesion, thus suggesting that conditions within the lesion environment that induce 
ER stress increase csGRP78 levels. This is further supported by increased expression of csGRP78 following treatment of HAECs with well-established lesion-resident agents/conditions known to elicit an ER stress response, including 7-ketocholesterol and peroxynitrite as well as csGRP78 expression on lesion-resident ECs (ref. 3 and Supplemental Figure 4).

To further understand the contribution of anti-GRP78 autoantibodies to atherogenesis, $A p o E^{-/-}$mice were immunized with rGRP78 to increase anti-GRP78 autoantibody titers. Six-week-old $A p o E^{-/-}$mice immunized against rGRP78 presented with higher anti-GRP78 autoantibody levels and had much larger lesions at 15 weeks of age compared with OVA-injected control mice. It is worth noting that both cohorts had similar plasma cholesterol and triglyceride levels, data that suggest that increased lesion size was not due to a change in circulating lipids. Mice with higher levels of anti-GRP78 autoantibodies also demonstrated a larger necrotic core area in lesions. Lipid-rich necrotic cores are considered a key feature of advanced lesions and correspond to increased instability of atherosclerotic plaques (37), suggesting that anti-GRP78 autoantibodies amplify lesion progression to a more complex and unstable state. Together, these findings suggest that anti-GRP78 autoantibodies exacerbate lesion development through interaction with lesion-resident ECs.

In this report, we demonstrate that anti-GRP78 autoantibody-mediated activation of csGRP78 stimulates expression of ICAM1 and VCAM1 as well as increased adhesion of monocytes to ECs. Based on these findings, we investigated two means of neutralizing the effect anti-GRP78 autoantibodies: a synthetic conformational peptide, CNVSKDSC, which mimics the N-terminal domain of GRP78 (15), and enoxaparin, a LMWH that can bind to GRP78 directly (38). Both approaches were effective in specifically neutralizing the effect of anti-GRP78 autoantibodies on increased expression of adhesion molecules. This observation confirms and provides a likely mechanism to explain the findings in previous studies reporting that LMWH intervention minimized the atherogenic diet-induced histopathological lesions in heart, liver, and kidney tissues of rats (39-41). Expression of adhesion molecules as well as the binding and transmigration of leukocytes into the subendothelial space are critical steps during the early stages of atherogenesis and are considered hallmarks of EC activation $(2,32)$. These findings strongly suggest that anti-GRP78 autoantibodies contribute to EC activation, lesion development, and progression.

Inflammation leads to recruitment of leukocytes to the surface of lesion-resident ECs, and the observed leukocyte/EC interactions, such as capture, rolling, and firm adhesion, can be viewed as a series of overlapping synergistic interactions among adhesion molecules, resulting in an adhesion cascade. These cascades direct leukocyte migration, which is essential for the generation of effective inflammatory responses and the development of rapid immune responses (30). The expression of the adhesion molecules is orchestrated by the NF- $\mathrm{kB}$ pathway; this is not only present in human atherosclerotic lesions, but also contributes to the pathogenesis of atherosclerosis $(42,43)$. Given that NF- $\mathrm{kB}$ has been shown to regulate the expression of adhesion molecules in ECs (31), we investigated the role of NF- $\mathrm{kB}$ based on the induction of adhesion molecule expression, including ICAM1 and VCAM1, induced by the autoantibodies. Expression of cytokines and chemokines occurs during

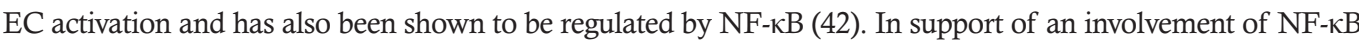
in anti-GRP78 autoantibody-mediated csGRP78 signaling, we observed increased expression of known proinflammatory mediators, activated by NF- $\mathrm{kB}$, in cells treated by anti-GRP78 autoantibodies. Further, we now demonstrate that EC activation by anti-GRP78 autoantibodies can be specifically reversed with either enoxaparin or NF- $\mathrm{kB}$ pathway inhibitors. Additional evidence supporting a connection between anti-GRP78 autoantibodies and NF- $\mathrm{KB}$ was recently described by Shimizu et al. (44) in brain microvascular ECs exposed to antibodies isolated from patients diagnosed with the autoimmune disorder neuromyelitis optica. Consistent with our experimental results, antibodies targeting csGRP78 activated NF- $\mathrm{BB}$ and induced ICAM1 expression; however, anti-GRP78 autoantibody titers were not determined in that study (44). Nevertheless, these findings emphasize that both anti-GRP78 autoantibodies and csGRP78 are required for NF- $\mathrm{BB}$ activation in lesion-resident ECs and this molecular mechanism contributes to lesion development and possibly plaque rupture.

Additional studies to identify the precise upstream molecular signals that connect NF- $\mathrm{BB}$ to anti-GRP78 autoantibody-stimulated csGRP78 signaling are actively being explored. Some potential pathways have been discussed here; however, their relevance in ECs remains to be established. Previous findings demonstrated an association between csGRP78 and a G protein complex at the plasma membrane of stimulated macrophages (45). It is known that activation of the heterotrimeric G protein leads to PKC activation, which in turn results in canonical IKK/NF- $\mathrm{KB}$ activation in ECs $(46,47)$. In addition, our group has demonstrated the ability of anti-GRP78 autoantibodies to activate cSGRP78 in bladder carcinoma cells, resulting in PLC-mediated release of $\mathrm{Ca}^{2+}$ from ER stores (8). Although the mechanism is not completely understood, it is thought 

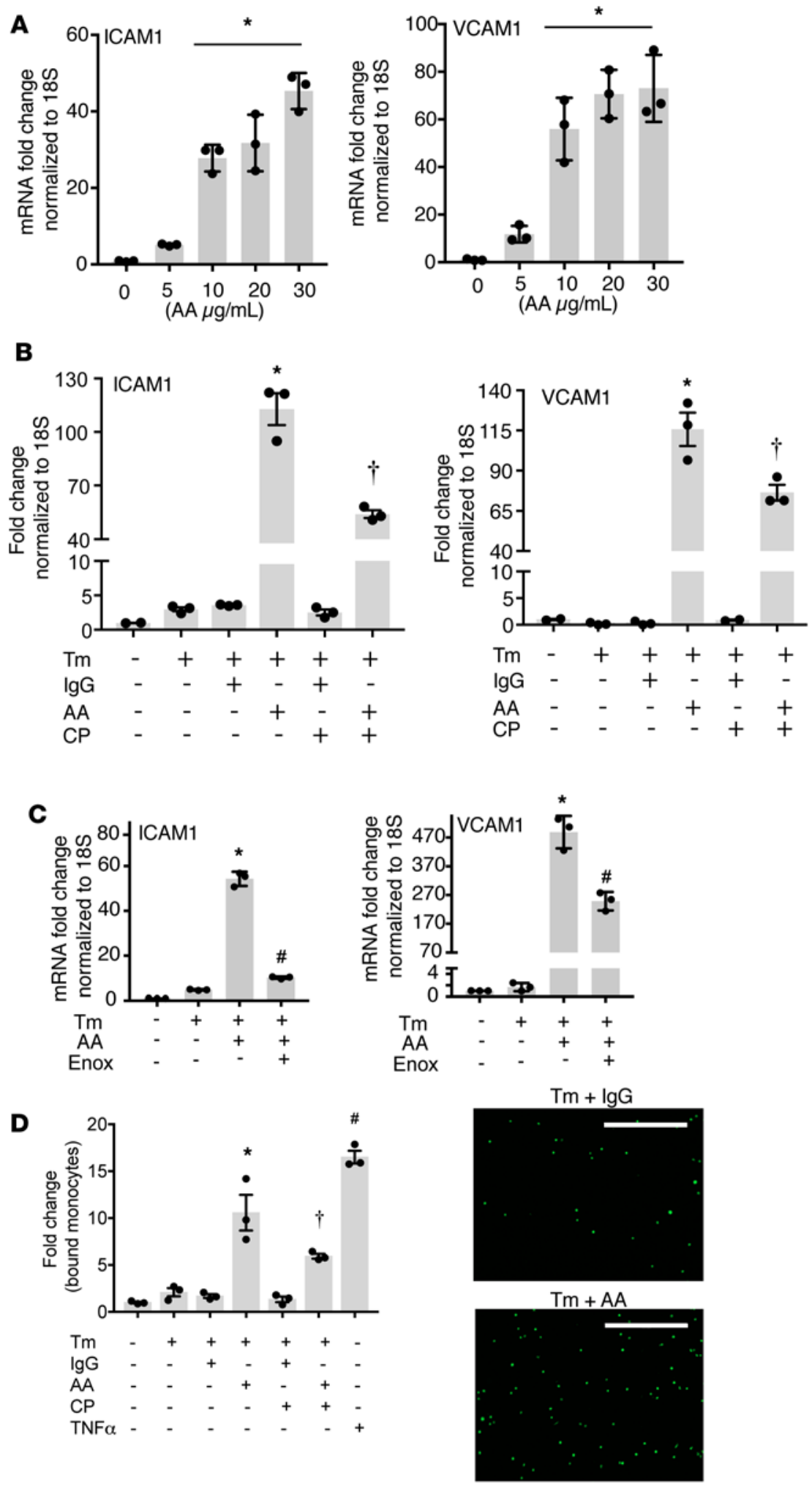

Figure 4. ER stress and anti-GRP78 autoantibodies synergistically enhance ICAM1 and VCAM1 expression. (A) Treatment of HAECs with increasing concentrations of anti-GRP78 autoantibody increases ICAM1 and VCAM1 mRNA expression, as measured by qRT-PCR. ${ }^{*} P<0.05$ versus untreated cells. Total RNA was isolated from HAECs pretreated without or with $2.5 \mu \mathrm{g} / \mathrm{ml} \mathrm{Tm}$ for 24 hours. Cells were then exposed to purified anti-GRP78 autoantibodies (AA, $60 \mu \mathrm{g}$ / $\mathrm{ml})$ or human IgG $(60 \mu \mathrm{g} / \mathrm{ml})$ for 8 hours without or with (B) CNVSKDSC peptide (CP, $90 \mu \mathrm{g} / \mathrm{ml})(\dagger P<$ 0.05 versus all other groups) or (C) enoxaparin (Enox, $50 \mu \mathrm{g} / \mathrm{ml})\left({ }^{*} P<0.05\right.$ versus nontreated cells). (D) Adhesion of BCECF-AM-loaded U937 cells to HAECs treated as described in $\mathbf{A}$, with representative images. Scale bar: $400 \mu \mathrm{m}$. HAECs treated with TNF- $\alpha$ (100 $\mathrm{ng} / \mathrm{ml}, 8$ hours) served as a positive control. Data are expressed as percentage adhesion relative to untreated cells ( $n=3$ per group). ${ }^{*} P<0.05$ versus all other groups. ${ }^{\dagger} P<0.05$ versus all other groups. ${ }^{\#} P<$ 0.05 versus all other groups. ANOVA and $t$ test were used for all statistical comparisons.

that an increase in cytosolic $\mathrm{Ca}^{2+}$ activates $\mathrm{NF}-\kappa \mathrm{B}$, possibly through production of mitochondrial reactive oxygen species $(48,49)$. Finally, recent studies utilizing therapeutically resistant cancer cells showed that csGRP78 complexes with PI3K as well as the ability of anti-GRP78 autoantibodies to induce csGRP78-driven PI3K-dependent AKT activation $(16,23)$. In ECs, NF- $\mathrm{KB}$ activation is known to be mediated by PI3K/AKT in response to TNF- $\alpha$ (50). Further work is currently being performed to characterize the molecular signals in ECs linking anti-GRP78 autoantibody/ csGRP78 circuit with NF- $\kappa B$ activation.

Chronic inflammation contributes to atherogenesis and CVD; a number of antibodies targeting self-antigens, such as autoantibodies against apolipoprotein A-1 (apoA-1) or oxidized LDL, have been associated with atherosclerosis, even in the absence of overt immunization or additional infection, leading to the theory that atherosclerosis could fundamentally be an autoimmune disease (51-54). Clinical evidence indicates that elevated levels of anti-apoA-1 antibodies correspond to a worse prognosis (55), supporting the idea that proinflammatory autoantibodies have a causal role in CVD. Similarly, high levels of anti-GRP78 autoantibodies in prostate cancer patients correlate with advanced disease and poor overall survival (15), although it remains to be determined whether the elevated levels of anti-GRP78 autoantibodies that we observed in patients with established CVD affect disease progression and plaque stability. Growing evidence supporting the role of autoantibodies in CVD and as a bona fide prognostic marker (56) highlights the need to identify new relevant targets, such as anti-GRP78 autoantibodies and to explore the potential therapeutic benefits of modulating their deleterious effects. 
A

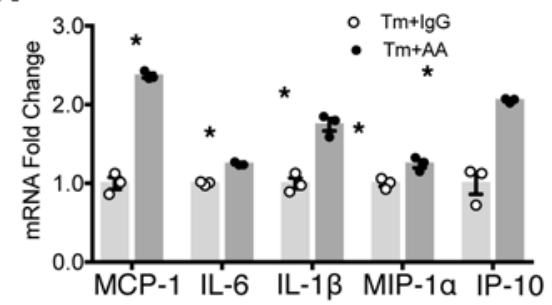

D

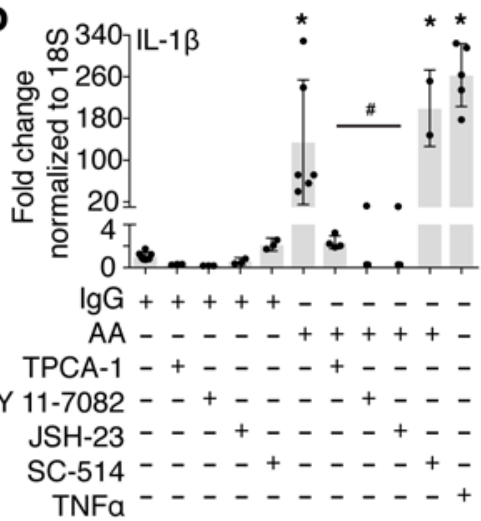

G

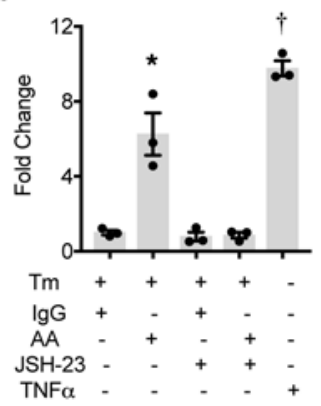

B

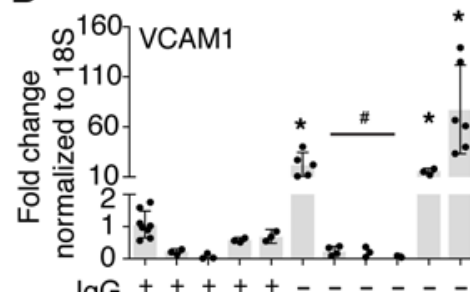

$\lg G+++++\ldots$

$\mathrm{AA}--\cdots+++$

TPCA-1 - + - - + - - -

BAY 11-7082 - + - - + + -

JSH-23 - - + - - - + +

$\mathrm{SC}-514$ - $-+\ldots+-+$

TNFa

$\mathbf{E}$

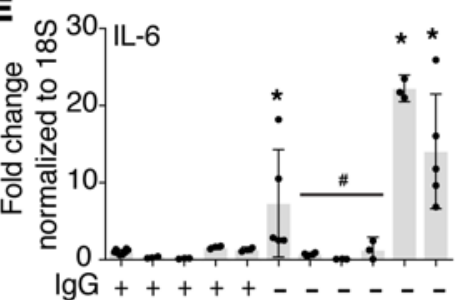

$\mathrm{AA}-{ }_{-}-+++++-$

TPCA-1 - + - - + - -

BAY 11-7082 - + + - + + -

JSH-23 - - + - - + + -

SC-514 _ - $+\ldots+-+$

TNFa - - - - - - - +

$\mathrm{Tm}+\mathrm{AA}$

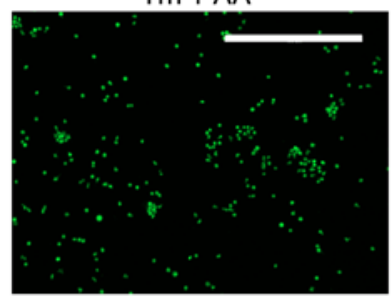

$\mathrm{Tm}+\mathrm{AA}+\mathrm{JSH}-23$

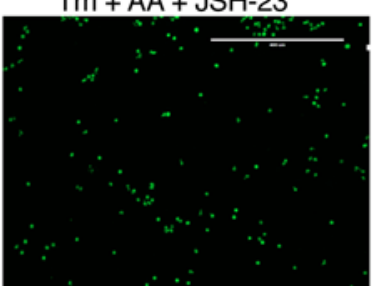

C

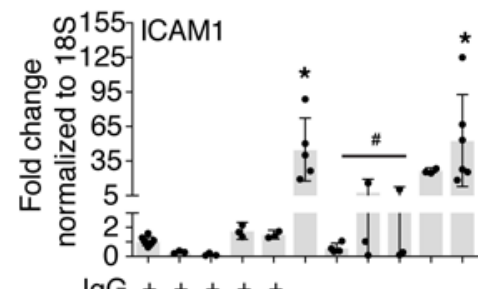

$\lg \mathrm{G}+++++\ldots$

$\mathrm{AA}-\mathrm{A}_{-}-++++$

TPCA-1 - + - - + - -

BAY 11-7082 - + + - + + - -

JSH-23 - - + - - + - -

SC-514 - - + + - - +

TNFa - - - - - - +

F

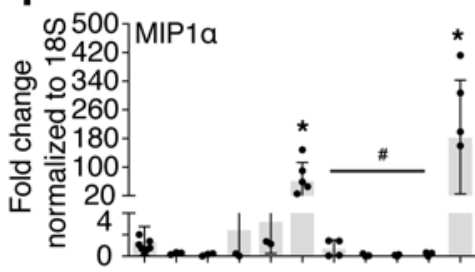

$\lg \mathrm{G}+++++\ldots$

$\mathrm{AA}$ - . - - + + + +

TPCA-1

BAY 11-7082 _ + + - - + - -

JSH-23 - - + - - + -

SC-514 - - - + - - +

TNFa _ . . . . . . +

Figure 5. Increased mRNA expression of ICAM1 and VCAM1 by anti-GRP78 autoantibodies in ECs is mediated by NF-кB. (A-F) Relative mRNA levels of cytokines and chemokines, ICAM1 and VCAM1, determined by qRT-PCR of HAECs treated with anti-GRP78 autoantibodies (AA, $60 \mu$ g/ml) or human IgC $(60 \mu \mathrm{g} / \mathrm{ml})$ and/or NF- $\mathrm{kB}$ inhibitors (TPCA-1, BAY 11-7082, JSH-23, SC-514). TNF- $\alpha$ was used as a positive control. Data are expressed as fold change relative to IgG-treated cells ( $n=3$ per group). ${ }^{*} P<0.05$ versus all other groups. ${ }^{\#} P<0.01$ versus anti-GRP78 autoantibody treatment alone. (C) Adhesion of BCECF-AM-loaded U937 cells to HAECs treated JSH-23 with or without anti-GRP78 autoantibodies. Representative images are shown. Scale bar: $400 \mu$ m. Data are expressed as percentage cell adhesion relative to Tm-treated cells ( $n=3$ per group). ${ }^{*} P<0.05$ versus all other groups. ${ }^{\dagger} P<0.05$ versus all other groups. ANOVA and $t$ test were used for all statistical comparisons.

Collectively, our results present a role for an anti-GRP78 autoantibody/csGRP78 signaling circuit in EC activation in early stages of atherosclerotic lesion development. To our knowledge, this work is the first to define a mechanism by which csGRP78 signaling accelerates atherogenesis. Translational strategies aimed at blocking csGRP78 signaling, possibly via the neutralization of circulating anti-GRP78 autoantibodies, have the potential of attenuating atherosclerotic lesion development in clinical applications. 
A

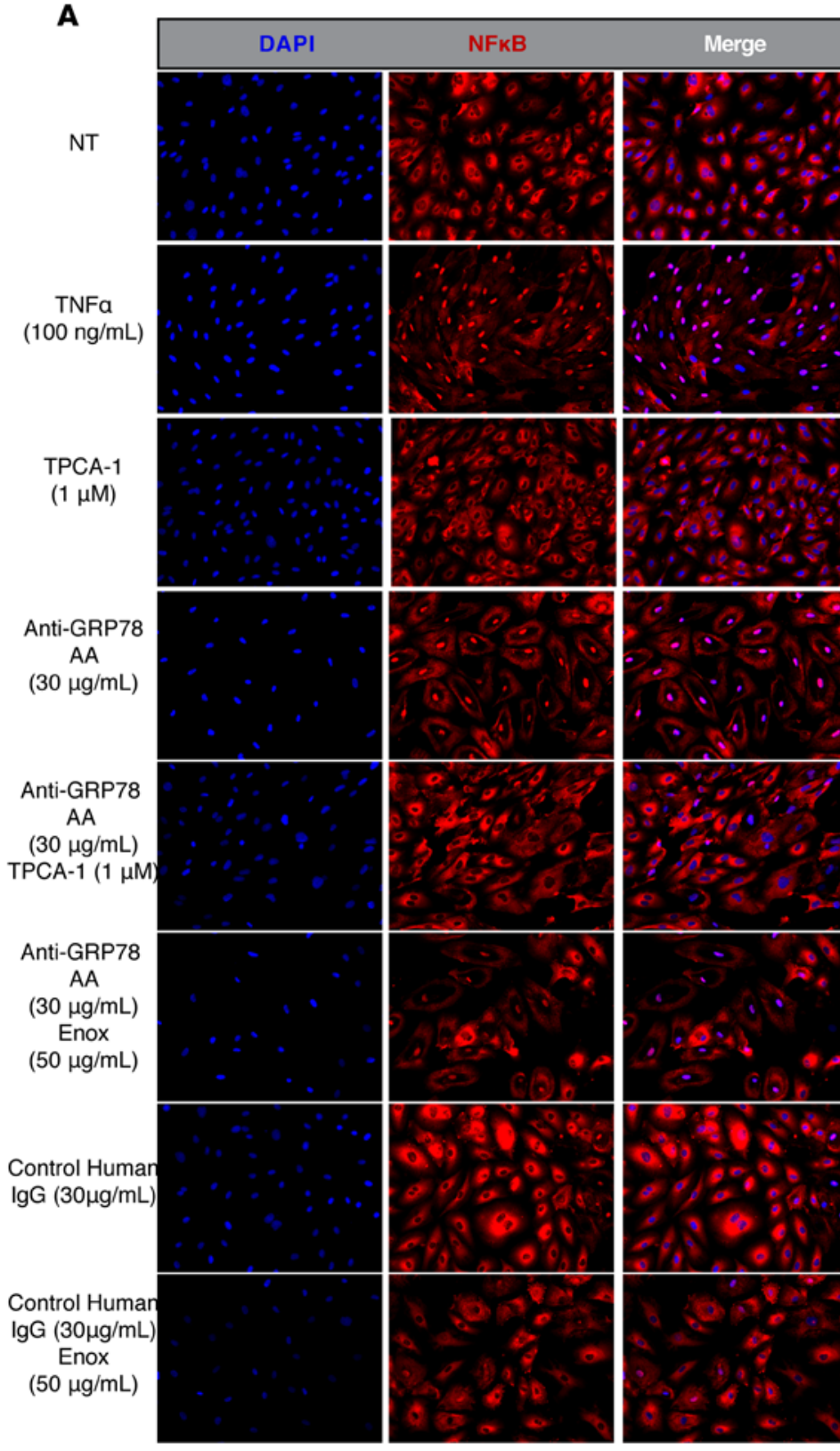

B

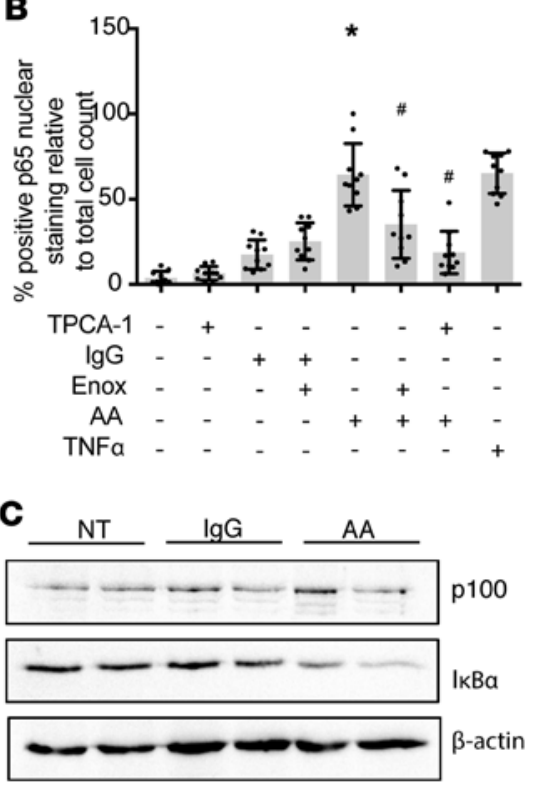

Quantification of western blot analysis
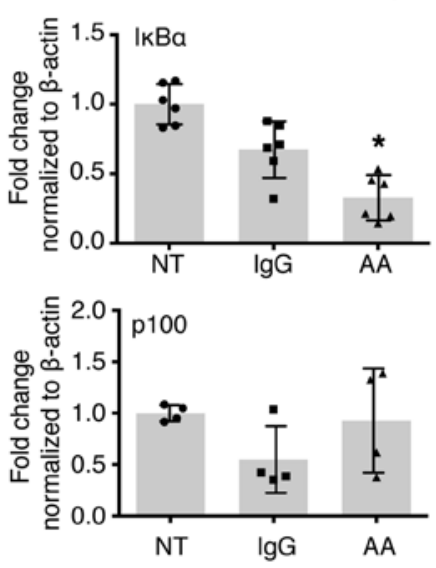

Figure 6. Anti-GRP78 autoantibodies induce NF-KB p65 nuclear localization. (A) HAECs were pretreated with either enoxaparin (Enox, $50 \mu \mathrm{g} / \mathrm{ml}$ ) or TPCA$1(1 \mu \mathrm{M})$ for 6 hours prior to treatment with either human IgG $(30 \mu \mathrm{g} / \mathrm{ml})$ or purified anti-CRP78 autoantibody (AA, $30 \mu \mathrm{g} / \mathrm{ml})$ for 12 hours. Representative images of immunofluorescence staining of NF- $\mathrm{KB}$ p65 subunit localization are presented. Original magnification, $\times 20$. (B) Quantification of positive $p 65$ nuclear staining. NF- $\kappa B$ p 65 is stained red (Cy5-conjugated secondary antibody). Nuclei were counterstained with DAPI ( $n=10$ per group). ${ }^{*} P<0.05$ versus IgG. ${ }^{\#} P<0.05$ versus anti-GRP78 autoantibody, ANOVA. (C) Anti-GRP78 autoantibody treatment substantially activates the NF- $\kappa B$ classical canonical pathway but not the alternative pathway, as determined by immunoblotting for IkB $\alpha$ and $p 100$, respectively. HAECs were treated with human IgG (IgG, 30 $\mu \mathrm{g} / \mathrm{ml}$ ) or purified anti-GRP78 autoantibodies (AA, $30 \mu \mathrm{g} / \mathrm{ml}$ ) for 6 hours. Total cell lysate were subjected to Western blotting for detection of p100 NF-KB subunit or IкB $\alpha$. Blots were also immunostained for $\beta$-actin as a protein loading control. Quantification of immunoblots is expressed as fold change. All values are represented as mean $\pm \mathrm{SD}$ ( $n=4-6$ per group). ${ }^{*} P<0.05$ versus IgG, 2 -tailed $t$ test.

\section{Methods}

Animals. Female C57BL/6 and $A p o E^{-/-}$mice from The Jackson Laboratory were fed either a normal chow or HFD ad libitum (Harlan Tekland; 88137), with free access to water. All mouse experiments were blinded to reduce observer's bias and were carried out in compliance with the McMaster Animal Research Ethics Board. In all experiments described here, blood was collected from the right ventricle under isoflurane anesthesia. Mice were then euthanized by cervical dislocation and immediately perfused with PBS followed by 
A
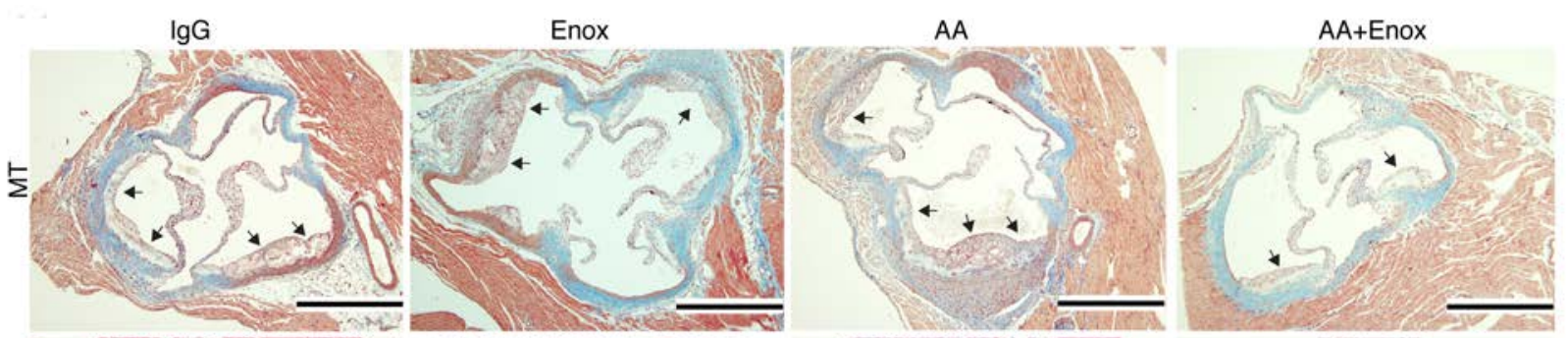

B
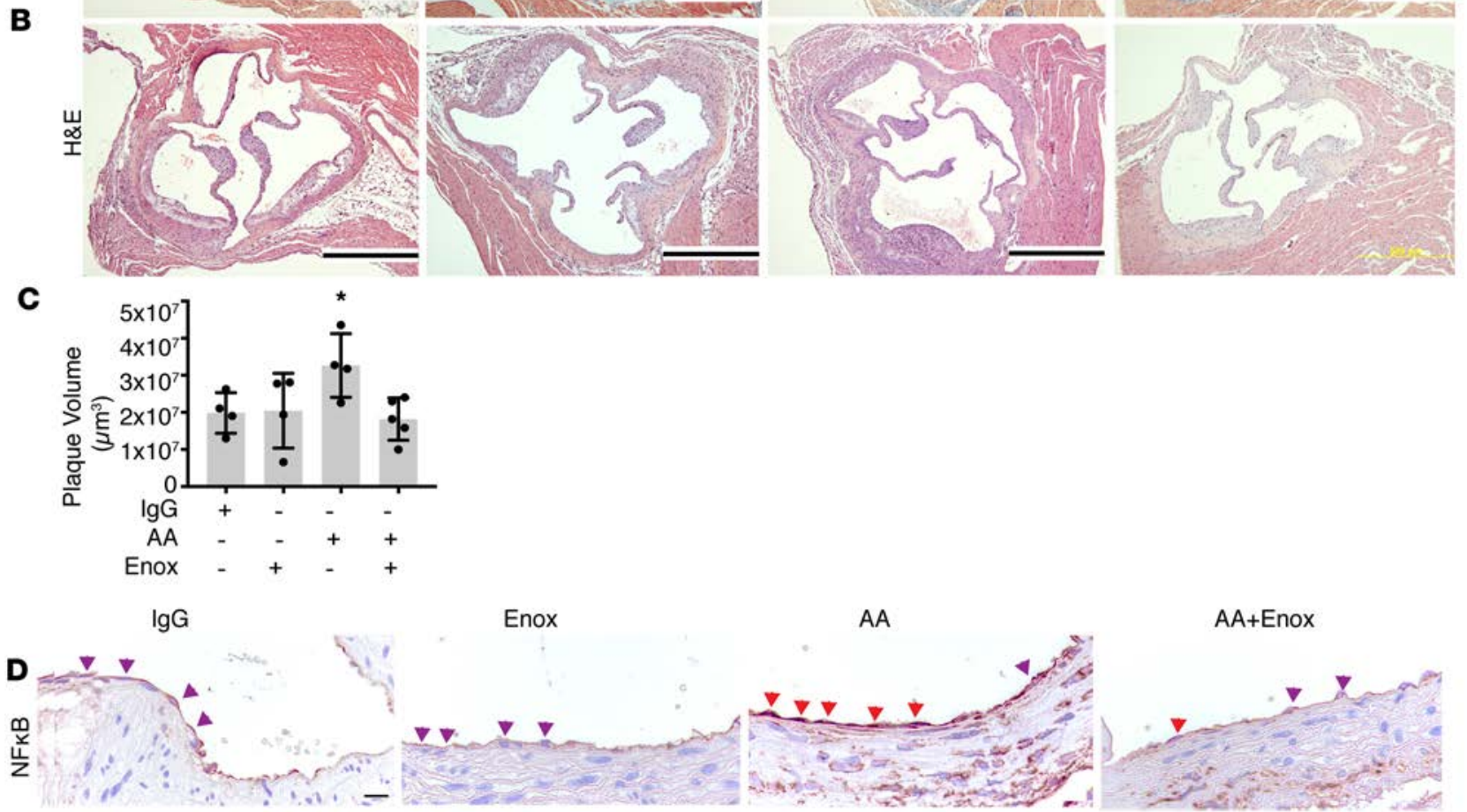

Figure 7. Enoxaparin intervention neutralizes the effect of anti-GRP78 autoantibody on accelerated atherosclerotic lesion growth in ApoE ${ }^{-/-}$mice. Female C57BL/ 6 or $\mathrm{ApoE}^{-/-}$mice on a chow diet were injected weekly for 5 weeks with control IgG (168 $\mu \mathrm{g}$ per mouse), anti-GRP78 autoantibody (168 $\mu \mathrm{g}$ per mouse), or enoxaparin (enox, $6 \mu \mathrm{g} / \mathrm{kg}$ ) ( $n=5$ per group). Representative images of mouse aortic roots stained with Mason's trichrome (MT) stain (A), hematoxylin/eosin (H\&E) (B), and NF-KB p65 nuclear stain (D). (C) Quantification of atherosclerotic lesion size at the aortic root from 15-week-old immunized mice. Lesion size was measured in 5-6 sections at $80 \mu \mathrm{m}$ intervals ( $n=4-5$ per group). ${ }^{*} P<0.01$ versus IgG treated mice, 2 -tailed $t$ test. Scale bar: $500 \mu \mathrm{m}$ (A and B); $50 \mu \mathrm{m}$ (D).

$4 \%$ paraformaldehyde. The heart and aorta were removed and fixed in buffered formalin overnight before further processing. To stimulate the production of anti-GRP78 autoantibodies, 6-week-old female ApoE $E^{-/-}$ mice fed a chow diet were injected with $100 \mu 1$ TiterMax Gold adjuvant emulsified in a 1:1 mixture with 50 mg endotoxin-free OVA or $50 \mathrm{mg}$ rGRP78 in sterile saline. Mice were given 2 booster injections at a 10-day interval. Blood samples were collected at intermediate time points from the submandibular vein. Mice were euthanized at 15 or 25 weeks of age as described above. Total serum cholesterol and triglycerides were measured with the enzymatic colorimetric assays Cholesterol E and L-Type Triglyceride M, respectively (Wako Diagnostics). Human rGRP78 was produced and purified in E. coli as described previously (8).

Cell culture. Primary HAECs were purchased from Clonetics and grown in endothelial growth medium-2 (EGM-2) supplemented with 1\% FBS and growth factors (EGM Bullet Kit, Lonza) at $37^{\circ} \mathrm{C}$ in $5 \% \mathrm{CO}_{2}$. Cells at passage 4-8 were treated with $300 \mathrm{nM}$ Tg (MilliporeSigma), $2.5 \mu \mathrm{g} / \mathrm{ml} \mathrm{Tm}$ (MilliporeSigma), $100 \mathrm{ng} / \mathrm{ml}$ TNF- $\alpha$ (R\&D Systems), 30 nM NF-кB Activation Inhibitor II (JSH-23; Santa-Cruz), and 60 mg/ml human anti-GRP78 autoantibodies; collected; and purified by affinity chromatography as previously described (8), or were treated with $60 \mathrm{mg} / \mathrm{ml}$ human IgG (MilliporeSigma) or the CNVSKDSC peptide (CanPeptide).

Western blotting. After treatments, HAECs were washed with cold PBS and total lysates were collected in SDS-PAGE lysis buffer (60 mM Tris-Cl, pH 6.8, 12.8\% glycerol, and 2.05\% SDS) containing protease inhibitors (Roche). Cell-surface proteins were biotinylated and isolated by the Pierce Cell Surface Protein Isolation Kit 


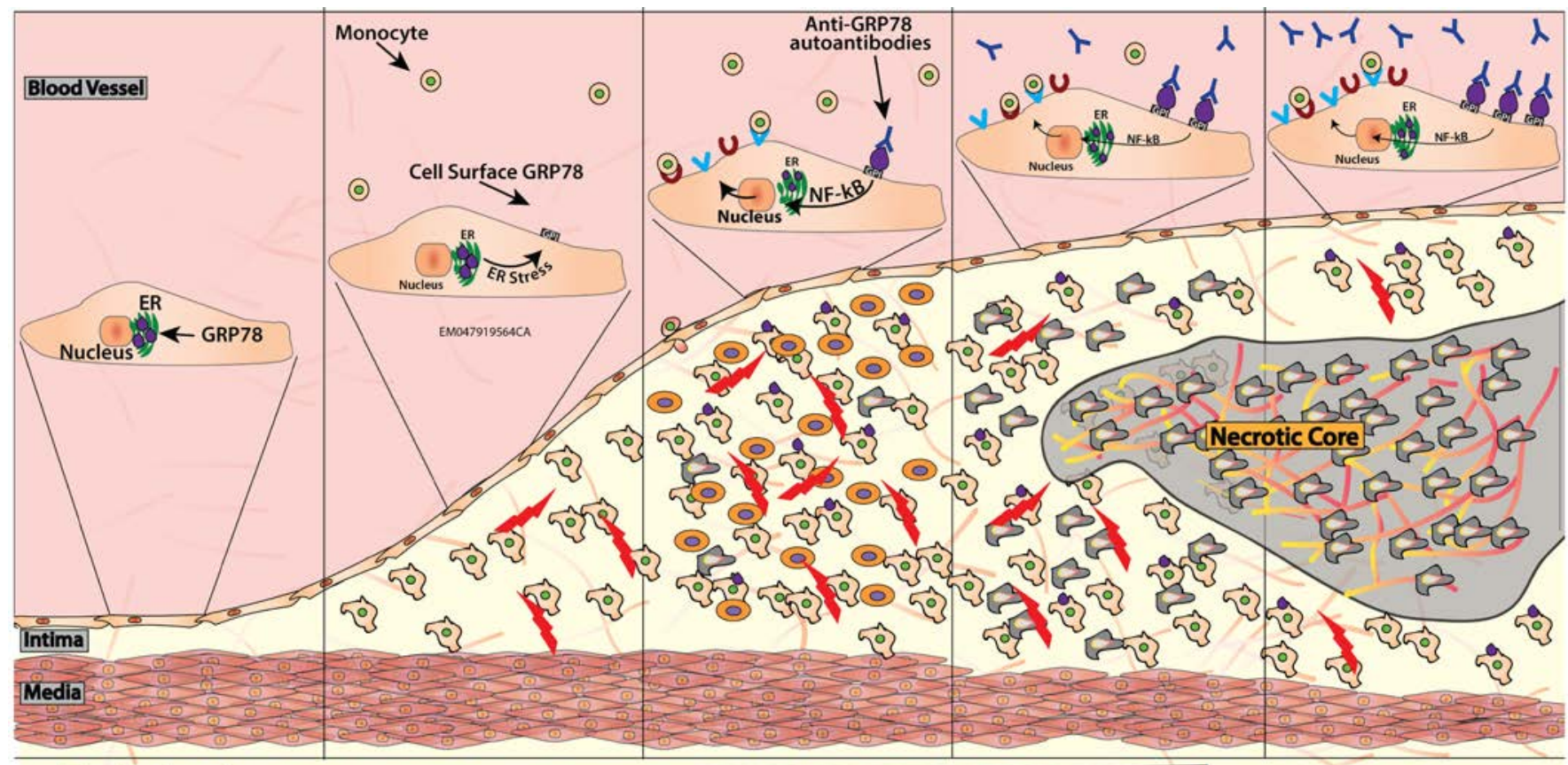

Early Lesion

Advanced Lesion

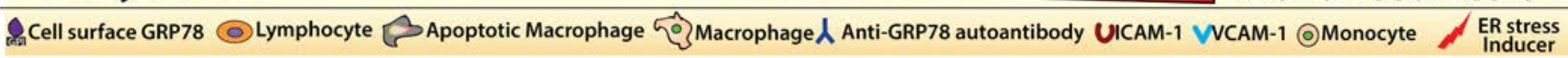

Figure 8. Schematic diagram illustrating the mechanism by which the binding of anti-GRP78 autoantibodies to csGRP78 on lesion-resident ECs accelerates atherosclerotic lesion growth via NF- $\mathrm{KB}$ activation. Conditions of ER stress in the early stages of atherosclerosis lead to the translocation of GRP78 from the ER to the cell surface. The presentation of csGRP78 induces an immunological response that drives the production of anti-GRP78 autoantibodies. Binding of anti-GRP78 autoantibodies to ECs activates the NF-KB pathway, leading to increased expression of adhesion molecules and the progression of atherosclerotic plaque.

(Thermo Scientific), as previously described (8). Total lysates and surface fractions were separated on $10 \%$ SDSPAGE mini-gels and transferred to nitrocellulose membranes. Membranes were incubated overnight at $4^{\circ} \mathrm{C}$ with the following antibodies: GRP78 (BD Transduction), CD31 (Cell Signaling), IRE-1 $\alpha$ (Cell Signaling), and $\beta$-actin (MilliporeSigma), followed by incubation with HRP-conjugated secondary antibodies (Bio-Rad) and visualization by Western Lighting Chemiluminescence Reagent (Perkin Elmer) and Kodak X-OMAT Blue XB-1 film.

Immunohistochemistry. Fixed hearts, including the aortic root, were dissected from the aortic arch, cut transversely, and paraffin embedded, as previously described (21). One hundred twenty-four-mm-thick serial sections were cut through the aortic root. Comparable sections were stained with H\&E. Lesion size and necrotic area were measured on $4-5$ sections at $80-\mu \mathrm{m}$ intervals using ImageJ $(\mathrm{NIH})$, as previously described (21). Necrotic area was expressed as a percentage of total lesion area. Aortic sections $(4-\mu \mathrm{m}$ thick) were deparaffinized, and the endogenous peroxidase activity was blocked with $0.5 \% \mathrm{H}_{2} \mathrm{O}_{2}$ in methanol for 10 minutes, as described previously (21). Following antigen retrieval where necessary, sections were blocked with 5\% normal serum, and incubated with the primary antibody for 1 hour, followed by biotinylated secondary antibody (Vector Laboratories, Burlingame, CA) diluted 1:500, and streptavidin HRP (Vector Laboratories) diluted 1:50. Sections were developed in Nova Red peroxidase substrate (Vector Laboratories) and counterstained with hematoxylin. Antibodies directed against NF- $\mathrm{kB}$ p65 (ab16502) or calreticulin (ab2907) were purchased from Abcam. Antibodies directed against GRP78 (sc-1050), CHOP (sc-575), or T cell death-associated gene 51 (TDAG51) (sc-6143) were from Santa Cruz Biotechnology. Rabbit anti-phospho-PERK (catalog 3191) was purchased from Cell Signaling. Conditions for antigen retrieval and dilution of antibodies have been described elsewhere (13).

RNA isolation and NanoString analysis. Total RNA was isolated from cells by using the RNeasy Tissue Mini Kit (Qiagen). For quality assurance, only samples with RNA integrity numbers greater than 5 were used for NanoString analysis. Data were normalized against 7 housekeeping genes, namely, IPO8, GUSB, TBP, YWHAZ, ACTB, GAPDH, and RPLP2. $P$ values were corrected for multiple comparisons with the Benjamini-Hochberg procedure by nSolver software (NanoString Technologies). 
$m R N A$ quantification by quantitative real-time PCR. Total RNA was isolated from HAECs with the RNeasy mini kit (Qiagen) and reverse-transcribed by using the High-Capacity cDNA Reverse Transcription kit (Applied Biosystems). Quantitative real-time PCR was performed in triplicate with Fast SYBR Green Master Mix (Applied Biosystems) under standard conditions. The primer sequences used are listed in Supplemental Table 1. $\beta 2$ Microglobulin (B2M) and 18S were used as housekeeping genes.

Analysis of serum anti-GRP78 autoantibody titers. Autoantibodies against GRP78 in mouse sera were determined by ELISA, as described previously (16). Our approach was to identify the antibodies recognizing the N-terminal of GRP78 by using a previously characterized synthetic peptide that mimics amino acids Leu $_{98}{ }^{-}$ Leu $_{115}$ in GRP78 (16). Briefly, 96-well plates were coated with the CNVSKDSC peptide conjugated to KLH ( $5 \mathrm{mg} / \mathrm{ml}$ ), blocked with PBS-Tween containing 3\% BSA, and incubated overnight with serum samples at a 1:100 dilution. Plates were incubated with anti-mouse IgG conjugated to alkaline phosphatase and developed with an alkaline phosphatase substrate. Absorbance was read at $405 \mathrm{~nm}$. All assays were performed in triplicate and analyzed relative to a standard sample to correct for plate-to-plate variation.

Cell adhesion assay. HAECs were seeded into 24-well fluorescence plates and allowed to adhere overnight. Cells were treated without or with $\mathrm{Tm}(2.5 \mathrm{mg} / \mathrm{ml}, 24$ hours $)$, followed by the indicated conditions. U937 cells (ATCC) were washed and resuspended at $1 \times 10^{6}$ cells $/ \mathrm{ml}$ in HBSS containing HEPES with BCECF-AM (4 mM, MilliporeSigma) for 30 minutes at room temperature. Cells were washed with growth media to remove excess fluorophore and resuspended at $1 \times 10^{6}$ cells $/ \mathrm{ml}$ in RPMI lacking phenol red (catalog 11835; Life Technologies). Treated HAECs were washed with PBS, and $50 \mu 1$ BCECF-AM-loaded U937 cells were added to each well. Fluorescence was measured at $439 \mathrm{~nm}$ excitation to determine the maximum signal for each well. After 30 minutes of incubation at $37^{\circ} \mathrm{C}, 3$ washes were performed to remove nonadherent cells. Fluorescence was measured a second time as described above. Adhesion was determined for each well as a percentage relative to the maximum fluorescence detected prior to washing. All assays were performed in triplicate.

En face immunofluorescence. Female $A p o E^{-/-}$mice at 18 weeks of age were injected i.v. in the tail vein with $10 \mathrm{mg}$ b-anti-GRP78 autoantibody or $10 \mathrm{mg}$ b-IgG. Mice were euthanized after 30 minutes as described above. The aortic arch was dissected and cut open longitudinally. The aortas were blocked with 10\% goat serum and incubated overnight with rat anti-CD31 (1:200; BD Pharmingen), followed by PBS washes and anti-rat Alexa Fluor 488 (1:200; Life Technologies) and streptavidin-conjugated Alexa Fluor 594 (1:200; Life Technologies) for 30 minutes. Nuclei were stained with DAPI (MilliporeSigma). The aortas were mounted on slides with ProLong Gold Antifade Mountant (Life Technologies) and imaged under a Zeiss Axioplan fluorescent microscope (Carl Zeiss Canada). Regions with and without lesions were identified based on published anatomical locations relative to the lesser and greater curvature area $(20,57)$.

Statistics. Values are expressed as mean \pm SD. Statistical analysis was performed by using an unpaired 2-tailed Student's $t$ test or ANOVA. When significance was attained using ANOVA, a Tukey's post hoc test was used to determine specific differences. Statistical significance was defined as $P<0.05$ unless otherwise specified.

Study approval. All animal studies and procedures were reviewed and approved by the McMaster University Animal Research Ethics Board (AUP 16-12-43). Patients were screened for CAD based on coronary $\mathrm{CT}$ angiogram. The average age of CAD and non-CAD participants was 62.7 and 53.8 years, respectively. This study was approved by the human Research Ethics Board at the University of Ottawa, Ottawa, Ontario, Canada (protocol 20160605).

\section{Author contributions}

EDC, AAAH, and RCA conceived and designed the experiments. EDC and AAAH conducted and analyzed the experiments, along with JC, EGL, KDW, ŠL, MN, KP, JHB, PL, BS, KR, JCK, KJR, SM, RP, WA, and RCA. RCA wrote the initial draft of the manuscript, to which all authors contributed edits and provided funding for the research.

\section{Acknowledgments}

This work was supported, in part, by research grants to RCA from the Heart and Stroke Foundation of Canada (G-15-0009389), the Canadian Institutes of Health Research (MOP-286787), Prostate Cancer Canada (D2017-1949), and a Heart and Stroke Foundation of Ontario Program Grant (PRG6502). Financial support from the Research Institute of St. Joe's Hamilton is acknowledged. RCA is a career investigator of the Heart and Stroke Foundation of Ontario and holds the Amgen Canada Research Chair in the Division 
of Nephrology at St. Joseph's Healthcare Hamilton and McMaster University. WA and RP have received support from the Gillson-Longenbaugh Foundation. We would like to thank Matthew Miller (McMaster Immunology Research Centre, McMaster University) for helpful discussions.

Address correspondence to: Richard C. Austin, Department of Medicine, Division of Nephrology, McMaster University and St. Joseph's Healthcare Hamilton, 50 Charlton Avenue East, Room T-3313, Hamilton, Ontario L8N 4A6, Canada. Phone: 905.522.1155 Ext. 35175; Email: austinr@taari.ca. Or to: Wadih Arap, Rutgers Cancer Institute of New Jersey and Division of Hematology/Oncology, Department of Medicine, Rutgers New Jersey Medical School, Newark, New Jersey 77103, USA. Phone: 973.972.0366; Email: wadih.arap@rutgers.edu.

1. Bijl M. Endothelial activation, endothelial dysfunction and premature atherosclerosis in systemic autoimmune diseases. Neth $J$ Med. 2003;61(9):273-277.

2. Ross R. Atherosclerosis is an inflammatory disease. Am Heart J. 1999;138(5 Pt 2):S419-S420.

3. Liu C, Bhattacharjee G, Boisvert W, Dilley R, Edgington T. In vivo interrogation of the molecular display of atherosclerotic lesion surfaces. Am J Pathol. 2003;163(5):1859-1871.

4. Gonzalez-Gronow M, Selim MA, Papalas J, Pizzo SV. GRP78: a multifunctional receptor on the cell surface. Antioxid Redox Signal. 2009;11(9):2299-2306

5. Ni M, Zhang Y, Lee AS. Beyond the endoplasmic reticulum: atypical GRP78 in cell viability, signalling and therapeutic targeting. Biochem J. 2011;434(2):181-188.

6. Misra UK, Deedwania R, Pizzo SV. Binding of activated alpha2-macroglobulin to its cell surface receptor GRP78 in 1-LN prostate cancer cells regulates PAK-2-dependent activation of LIMK. J Biol Chem. 2005;280(28):26278-26286.

7. Burikhanov R, Zhao Y, Goswami A, Qiu S, Schwarze SR, Rangnekar VM. The tumor suppressor Par-4 activates an extrinsic pathway for apoptosis. Cell. 2009;138(2):377-388.

8. Al-Hashimi AA, et al. Binding of anti-GRP78 autoantibodies to cell surface GRP78 increases tissue factor procoagulant activity via the release of calcium from endoplasmic reticulum stores. J Biol Chem. 2010;285(37):28912-28923.

9. Al-Hashimi AA, et al. Autoantibodies against the cell surface-associated chaperone GRP78 stimulate tumor growth via tissue factor. J Biol Chem. 2017;292(51):21180-21192.

10. Tsai YL, Zhang Y, Tseng CC, Stanciauskas R, Pinaud F, Lee AS. Characterization and mechanism of stress-induced translocation of 78-kilodalton glucose-regulated protein (GRP78) to the cell surface. J Biol Chem. 2015;290(13):8049-8064.

11. Zhang Y, Liu R, Ni M, Gill P, Lee AS. Cell surface relocalization of the endoplasmic reticulum chaperone and unfolded protein response regulator GRP78/BiP. J Biol Chem. 2010;285(20):15065-15075.

12. Zhou J, et al. Association of multiple cellular stress pathways with accelerated atherosclerosis in hyperhomocysteinemic apolipoprotein E-deficient mice. Circulation. 2004;110(2):207-213.

13. Zhou J, Lhoták S, Hilditch BA, Austin RC. Activation of the unfolded protein response occurs at all stages of atherosclerotic lesion development in apolipoprotein E-deficient mice. Circulation. 2005;111(14):1814-1821.

14. Tabas I, García-Cardeña G, Owens GK. Recent insights into the cellular biology of atherosclerosis. J Cell Biol. 2015;209(1):13-22

15. Mintz PJ, et al. Fingerprinting the circulating repertoire of antibodies from cancer patients. Nat Biotechnol. 2003;21(1):57-63.

16. Gonzalez-Gronow M, Cuchacovich M, Llanos C, Urzua C, Gawdi G, Pizzo SV. Prostate cancer cell proliferation in vitro is modulated by antibodies against glucose-regulated protein 78 isolated from patient serum. Cancer Res. 2006;66(23):11424-11431.

17. Dharmarajan TS, Sohagia A. Low-molecular-weight heparin and bleeding: how do we lower risk but maintain benefit? Ann Intern Med. 2006;145(10):789-790.

18. Basseri S, Lhoták S, Sharma AM, Austin RC. The chemical chaperone 4-phenylbutyrate inhibits adipogenesis by modulating the unfolded protein response. J Lipid Res. 2009;50(12):2486-2501.

19. Erbay E, et al. Reducing endoplasmic reticulum stress through a macrophage lipid chaperone alleviates atherosclerosis. Nat Med. 2009;15(12):1383-1391.

20. Iiyama K, et al. Patterns of vascular cell adhesion molecule-1 and intercellular adhesion molecule-1 expression in rabbit and mouse atherosclerotic lesions and at sites predisposed to lesion formation. Circ Res. 1999;85(2):199-207.

21. Lhoták S, Zhou J, Austin RC. Immunohistochemical detection of the unfolded protein response in atherosclerotic plaques. Meth Enzymol. 2011;489:23-46.

22. van Vlijmen BJ, et al. Diet-induced hyperlipoproteinemia and atherosclerosis in apolipoprotein E3-Leiden transgenic mice. $J$ Clin Invest. 1994;93(4):1403-1410.

23. Zhang Y, Tseng CC, Tsai YL, Fu X, Schiff R, Lee AS. Cancer cells resistant to therapy promote cell surface relocalization of GRP78 which complexes with PI3K and enhances PI(3,4,5)P3 production. PLoS ONE. 2013;8(11):e80071.

24. Bouvier N, et al. Cyclosporine triggers endoplasmic reticulum stress in endothelial cells: a role for endothelial phenotypic chang es and death. Am J Physiol Renal Physiol. 2009;296(1):F160-F169.

25. Sanson M, et al. Oxidized low-density lipoproteins trigger endoplasmic reticulum stress in vascular cells: prevention by oxygen-regulated protein 150 expression. Circ Res. 2009;104(3):328-336.

26. Dickhout JG, Hossain GS, Pozza LM, Zhou J, Lhoták S, Austin RC. Peroxynitrite causes endoplasmic reticulum stress and apoptosis in human vascular endothelium: implications in atherogenesis. Arterioscler Thromb Vasc Biol. 2005;25(12):2623-2629.

27. Mousa SA, Linhardt R, Francis JL, Amirkhosravi A. Anti-metastatic effect of a non-anticoagulant low-molecular-weight heparin versus the standard low-molecular-weight heparin, enoxaparin. Thromb Haemost. 2006;96(6):816-821.

28. Collins RG, Velji R, Guevara NV, Hicks MJ, Chan L, Beaudet AL. P-Selectin or intercellular adhesion molecule (ICAM)-1 deficiency substantially protects against atherosclerosis in apolipoprotein E-deficient mice. J Exp Med. 2000;191(1):189-194 
29. Cybulsky MI, et al. A major role for VCAM-1, but not ICAM-1, in early atherosclerosis. J Clin Invest. 2001;107(10):1255-1262.

30. Golias C, Tsoutsi E, Matziridis A, Makridis P, Batistatou A, Charalabopoulos K. Review. Leukocyte and endothelial cell adhesion molecules in inflammation focusing on inflammatory heart disease. In Vivo. 2007;21(5):757-769.

31. Zhou Z, Connell MC, MacEwan DJ. TNFR1-induced NF-kappaB, but not ERK, p38MAPK or JNK activation, mediates TNF-induced ICAM-1 and VCAM-1 expression on endothelial cells. Cell Signal. 2007;19(6):1238-1248.

32. Libby P, Hansson GK. Inflammation and immunity in diseases of the arterial tree: players and layers. Circ Res. 2015;116(2):307-311.

33. Lewis MJ, Pelham HR. Ligand-induced redistribution of a human KDEL receptor from the Golgi complex to the endoplasmic reticulum. Cell. 1992;68(2):353-364.

34. Orci L, et al. Bidirectional transport by distinct populations of COPI-coated vesicles. Cell. 1997;90(2):335-349.

35. Llewellyn DH, Roderick HL, Rose S. KDEL receptor expression is not coordinatedly up-regulated with ER stress-induced reticuloplasmin expression in HeLa cells. Biochem Biophys Res Commun. 1997;240(1):36-40.

36. Yamamoto K, et al. The KDEL receptor mediates a retrieval mechanism that contributes to quality control at the endoplasmic reticulum. EMBO J. 2001;20(12):3082-3091.

37. Libby P, et al. Macrophages and atherosclerotic plaque stability. Curr Opin Lipidol. 1996;7(5):330-335.

38. Hansen LK, O'Leary JJ, Skubitz AP, Furcht LT, McCarthy JB. Identification of a homologous heparin binding peptide sequence present in fibronectin and the $70 \mathrm{kDa}$ family of heat-shock proteins. Biochim Biophys Acta. 1995;1252(1):135-145.

39. Deepa PR, Varalakshmi P. Protective effects of certoparin sodium, a low molecular weight heparin derivative, in experimental atherosclerosis. Clin Chim Acta. 2004;339(1-2):105-115.

40. Artico M, et al. Protective role of parnaparin in reducing systemic inflammation and atherosclerotic plaque formation in ApoE/- mice. Int J Mol Med. 2011;27(4):561-565.

41. Bonomini F, et al. Role of parnaparin in atherosclerosis. Int J Exp Pathol. 2016;97(6):457-464.

42. de Winther MP, Kanters E, Kraal G, Hofker MH. Nuclear factor kappaB signaling in atherogenesis. Arterioscler Thromb Vasc Biol. 2005;25(5):904-914.

43. Brand K, et al. Activated transcription factor nuclear factor-kappa B is present in the atherosclerotic lesion. J Clin Invest. 1996;97(7):1715-1722.

44. Shimizu F, et al. Glucose-regulated protein 78 autoantibody associates with blood-brain barrier disruption in neuromyelitis optica. Sci Transl Med. 2017;9(397):eaai9111.

45. Misra UK, Pizzo SV. Heterotrimeric Galphaq11 co-immunoprecipitates with surface-anchored GRP78 from plasma membranes of alpha2M*-stimulated macrophages. J Cell Biochem. 2008;104(1):96-104.

46. Bair AM, et al. Ca2+ entry via TRPC channels is necessary for thrombin-induced NF-kappaB activation in endothelial cells through AMP-activated protein kinase and protein kinase Cdelta. J Biol Chem. 2009;284(1):563-574.

47. Rahman A, Fazal F. Blocking NF-кB: an inflammatory issue. Proc Am Thorac Soc. 2011;8(6):497-503.

48. Pahl HL, Baeuerle PA. A novel signal transduction pathway from the endoplasmic reticulum to the nucleus is mediated by transcription factor NF-kappa B. EMBO J. 1995;14(11):2580-2588.

49. Hawkins BJ, et al. G protein-coupled receptor Ca2+-linked mitochondrial reactive oxygen species are essential for endothelial/ leukocyte adherence. Mol Cell Biol. 2007;27(21):7582-7593.

50. Frey RS, Gao X, Javaid K, Siddiqui SS, Rahman A, Malik AB. Phosphatidylinositol 3-kinase gamma signaling through protein kinase Czeta induces NADPH oxidase-mediated oxidant generation and NF-kappaB activation in endothelial cells. $J$ Biol Chem. 2006;281(23):16128-16138.

51. Vuilleumier N, Montecucco F, Hartley O. Autoantibodies to apolipoprotein A-1 as a biomarker of cardiovascular autoimmunity World J Cardiol. 2014;6(5):314-326.

52. Ylä-Herttuala S, Palinski W, Butler SW, Picard S, Steinberg D, Witztum JL. Rabbit and human atherosclerotic lesions contain IgG that recognizes epitopes of oxidized LDL. Arterioscler Thromb. 1994;14(1):32-40.

53. Binder CJ, et al. The role of natural antibodies in atherogenesis. J Lipid Res. 2005;46(7):1353-1363.

54. Blasi C. The autoimmune origin of atherosclerosis. Atherosclerosis. 2008;201(1):17-32.

55. Vuilleumier N, et al. Anti-apolipoprotein A-1 IgG as an independent cardiovascular prognostic marker affecting basal heart rate in myocardial infarction. Eur Heart J. 2010;31(7):815-823.

56. Roux-Lombard P, Pagano S, Montecucco F, Satta N, Vuilleumier N. Auto-antibodies as emergent prognostic markers and possible mediators of ischemic cardiovascular diseases. Clin Rev Allergy Immunol. 2013;44(1):84-97.

57. Hajra L, Evans AI, Chen M, Hyduk SJ, Collins T, Cybulsky MI. The NF-kappa B signal transduction pathway in aortic endothelial cells is primed for activation in regions predisposed to atherosclerotic lesion formation. Proc Natl Acad Sci USA 2000;97(16):9052-9057. 